Article

\title{
The Health-Related Quality of Life of Sarcoma Patients and Survivors in Germany-Cross-Sectional Results of a Nationwide Observational Study (PROSa)
}

\author{
Martin Eichler ${ }^{1,2, * \mathbb{C}}$, Leopold Hentschel ${ }^{2}$, Stephan Richter ${ }^{1}$, Peter Hohenberger ${ }^{3}$, \\ Bernd Kasper ${ }^{4}\left(\mathbb{D}\right.$, Dimosthenis Andreou ${ }^{5,6} \oplus^{-}$, Daniel Pink ${ }^{6,7}{ }^{\circ}$, Jens Jakob ${ }^{8}$, Susanne Singer ${ }^{9}{ }^{\circ}$, \\ Robert Grützmann ${ }^{10}$, Stephen Fung ${ }^{11}$, Eva Wardelmann ${ }^{12}$, Karin Arndt ${ }^{13}$, Vitali Heidt ${ }^{14}$, \\ Christine Hofbauer ${ }^{2,15}$, Marius Fried ${ }^{16}$, Verena I. Gaidzik ${ }^{17}$, Karl Verpoort ${ }^{18}$, Marit Ahrens ${ }^{19}$, \\ Jürgen Weitz ${ }^{2,20}$, Klaus-Dieter Schaser 2,15, Martin Bornhäuser 1,2, Jochen Schmitt ${ }^{2,21}$, \\ Markus K. Schuler ${ }^{1,22}$ (1) and the PROSa Study Group ${ }^{\dagger}$
}

1 Clinic and Polyclinic for Internal Medicine I, University Hospital Carl Gustav Carus, TU Dresden, 01307 Dresden, Germany; stephan.richter@uniklinikum-dresden.de (S.R.); martin.bornhaeuser@uniklinikum-dresden.de (M.B.); markus.schuler@helios-gesundheit.de (M.K.S.)

2 National Center for Tumor Diseases (NCT/UCC), 01307 Dresden, Germany; Leopold.Hentschel@uniklinikum-dresden.de (L.H.); christine.hofbauer@uniklinikum-dresden.de (C.H.); juergen.weitz@uniklinikum-dresden.de (J.W.); Klaus-Dieter.Schaser@uniklinikum-dresden.de (K.-D.S.); jochen.schmitt@uniklinikum-dresden.de (J.S.)

3 Division of Surgical Oncology \& Thoracic Surgery, Mannheim University Medical Center, University of Heidelberg, 68167 Mannheim, Germany; peter.hohenberger@umm.de

4 Interdisciplinary Tumor Center, Sarcoma Unit, University Medical Center Mannheim, 68167 Mannheim, Germany; bernd.kasper@umm.de

5 Department of General Orthopedics and Tumor Orthopedics, University Hospital Munster, 48149 Munster, Germany; Dimosthenis.andreou@helios-gesundheit.de

6 Sarcoma Center Berlin-Brandenburg, Helios Hospital Bad Saarow, 15526 Bad Saarow, Germany; daniel.pink@helios-kliniken.de

7 Department of Internal Medicine C, University Hospital Greifswald, 17475 Greifswald, Germany

8 Clinic for General, Visceral, and Pediatric Surgery, University Hospital Goettingen, 37075 Goettingen, Germany; jens.jakob@med.uni-goettingen.de

9 Institute for Medical Biostatistics, Epidemiology and Informatic, University Hospital Mainz, 55131 Mainz, Germany; singers@uni-mainz.de

10 Clinic for Surgery, University Hospital Erlangen, 91054 Erlangen, Germany; Robert.Gruetzmann@uk-erlangen.de

11 Clinic for General, Visceral, and Pediatric Surgery, University Hospital Dusseldorf, 40225 Dusseldorf, Germany; Stephen.Fung@med.uni-duesseldorf.de

12 Gerhard-Domagk-Institute for Pathology, University Hospital Munster, 48149 Munster, Germany; eva.wardelmann@ukmuenster.de

13 German Sarcoma Foundation, 61200 Woelfersheim, Germany; Karin.Arndt@sarkome.de

14 The Scientific Institute of Office-based Hematologists and Oncologists, 50676 Cologne, Germany; heidt@winho.de

15 University Center for Orthopedics and Trauma Surgery, TU Dresden, 01307 Dresden, Germany

16 Clinic and Polyclinic for Internal Medicine III/University-Centre for Tumor Diseases,

University Hospital Mainz, 55131 Mainz, Germany; Marius.Fried@unimedizin-mainz.de

17 Clinic for Internal Medicine III, University Hospital Ulm, 89081 Ulm, Germany; Verena.Gaidzik@uniklinik-ulm.de

18 Inter-Local Joint Practice, Dres. Verpoort, Wierecky \& Brandl, 20259 Hamburg, Germany; k.verpoort@t-online.de

19 Medical Clinic II, University Hospital Frankfurt, 60590 Frankfurt am Main, Germany; marit.ahrens@kgu.de

20 Department of Visceral, Thoracic and Vascular Surgery, University Hospital Carl Gustav Carus, TU Dresden, 01307 Dresden, Germany

21 Center for Evidence-Based Healthcare, University Hospital Carl Gustav Carus, Technical University Dresden, 01307 Dresden, Germany 
22 Helios Hospital Emil von Behring, Department of Oncology, 14165 Berlin, Germany

* Correspondence: martin.eichler@uniklinikum-dresden.de; Tel.: +49-(0)351-19057

+ The PROSa study group consists of an expert advisory board, which includes representatives from the fields of sarcoma treatment and diagnosis, patient representatives and physicians from the participating office based practices and clinics.

Received: 3 November 2020; Accepted: 27 November 2020; Published: 30 November 2020

Simple Summary: Sarcomas are a rare cancer with many different subtypes. They can occur anywhere in the body and are treated in a multi-disciplinary manner. Large studies on the quality of life of sarcoma patients are rare, so little is known about how patients are doing compared to the general population and which groups of sarcoma patients are particularly affected by quality of life limitations. We assessed the quality of life of 1113 sarcoma patients from Germany. The majority were particularly restricted in their emotional functioning, physical functioning, and the exercise of everyday demands (role function). Many of them experienced pain (56\%) and fatigue (51\%). We found that patients with leg or bone sarcomas were especially affected by quality of life limitations. We also found that patients who received a retirement pension were less affected by quality of life restrictions than patients who had not retired.

Abstract: Sarcomas are rare cancers with high heterogeneity in terms of type, location, and treatment. The health-related quality of life (HRQoL) of sarcoma patients has rarely been investigated and is the subject of this analysis. Adult sarcoma patients and survivors were assessed between September 2017 and February 2019 in 39 study centers in Germany using standardized, validated questionnaires (European Organization for Research and Treatment of Cancer Quality of Life Questionnaire (EORTC QLQ-C30)). Associated factors were analyzed exploratively using multivariable linear regressions. Among 1113 patients, clinically important limitations and symptoms were most pronounced in emotional (63\%, 95\% CI 60-66\%), physical (60\%, 95\% CI 57-62\%), role functioning $(51 \%, 95 \%$ CI $48-54 \%)$, and pain (56\%, 95\% CI 53-59\%) and fatigue (51\%, 95\% CI $48-54 \%)$. HRQoL differed between tumor locations with lower extremities performing the worst and sarcoma types with bone sarcoma types being most affected. Additionally, female gender, higher age, lower socioeconomic status, recurrent disease, not being in retirement, comorbidities, and being in treatment were associated with lower HRQoL. Sarcoma patients are severely restricted in their HRQoL, especially in functioning scales. The heterogeneity of sarcomas with regard to type and location is reflected in HRQoL outcomes. During treatment and follow-up, close attention has to be paid to the reintegration of the patients into daily life as well as to their physical abilities and emotional distress.

Keywords: sarcoma; health-related quality of life; rare disease; observational study; clinically important restrictions and symptoms

\section{Introduction}

Sarcomas are rare cancers, with about 7000 new cases per year in Germany [1] and an incidence of around 5 per 100,000 in Europe [2]. Five-year relative survival in 2000-2002 was 58\% for soft tissue sarcomas and $62 \%$ for bone sarcomas [2]. Sarcomas form a heterogeneous group of tumors that includes a large variety of over 100 histological subtypes [3], can occur anywhere on the body, and whose therapy is based on complex and divergent treatment algorithms [4]. Preferred treatment modality for localized soft tissue sarcomas is surgery, often combined with (neo)adjuvant radiotherapy and/or (neo)adjuvant chemotherapy, depending on a variety of factors like tumor grade, histology and tumor location [4]. For bone sarcomas surgery is the first choice of treatment as well, in a variety of 
cases combined with chemotherapy and/ or radiotherapy [5]. If sarcomas are clinically not respectable radiotherapy or radiochemotherapy is used [4]. For gastrointestinal stromal tumors (GIST) surgery and a variety of tyrosine kinase inhibitors (TKI) are the preferred treatment options, depending on type of mutation, tumor size and other factors [6]. For locally advanced or marginally resectable extremity soft tissue sarcomas isolated limb perfusion (ILP) can be considered [7]. Regional hyperthermia is an option for localized high-risk soft tissue sarcomas in addition to neoadjuvant chemotherapy [8]. Un-resectable metastatic sarcomas are often treated with palliative chemotherapy and/or radiotherapy [4].

Sarcomas are often diagnosed late due to unspecific symptoms and rare occurrence [9]. Unplanned resections, result of misdiagnosing the tumor as a more common benign lesion, with a negative influence on the course of treatment are common [10,11]. Since 2018 in in Germany, it has been possible to have sarcoma centers certified as modules of an oncology center by the German Cancer Society [12]. Treatment at specialized centers is recommended by international guidelines [13]. In 2019, the "German Sarcoma Foundation" was founded, which is a joint organization of patients and physicians that is committed to improving the situation for sarcoma patients [14]. In 2017, the European Reference Network EURACAN (European Reference Network on Rare Adult Cancers (solid tumors)) for rare solid tumors in adults was established [15].

In addition to prolonged survival, cancer patients rate the improvement of quality of life as an important criterion for the treatment of tumor diseases [16,17]. However, the health-related quality of life (HRQoL) of sarcoma patients in the different stages of the disease is a rarely investigated topic worldwide [18]. This may be due to the rarity of the disease and the fact that sarcoma patients are treated at different facilities. A systematic review found a total of 20 publications between 2007 and 2017 [19]. The available publications refer to drug studies [20,21], specific localizations/ entities [22-24], single disease phases [25-27], or have small sample sizes [28]. The large study by van Eck et al. focused on the heterogeneity of surviving sarcoma patients in terms of tumor location [29].

This resulted in the following questions, which we addressed in an exploratory analysis:

(1) How is the HRQoL of sarcoma patients in Germany? How high is the percentage of patients with clinically important limitations and symptoms in the individual domains of HRQoL?

(2) Which factors are associated with selected HRQoL domains? Are there differences between sarcoma subtypes with respect to histology and location?

\section{Results}

\subsection{Participation and Sample Description}

Approximately 1900 patients and survivors were approached and 1309 participated in the study (participation rate estimate: 69\%). HRQoL data were available for 1113 patients and survivors (Figure 1). $70 \%$ of participants with HRQoL data had soft tissue sarcoma, $18 \%$ had bone sarcoma, and $12 \%$ had GIST. 33\% of patients were under treatment (Table 1).

\subsection{Health-Related Quality of Life}

Mean global HRQoL was 59.5 out of a maximum of 100 points (Standard Deviation (SD) 22.7). Among the functioning scales, social (57.9, SD 33.1) and role functioning (54.3, SD 33.6) had the lowest values. Fatigue (43.2, SD 28.5), insomnia (38.5, SD 34.1), and pain (34.1, SD 31.6) showed the highest symptom loads. Gastrointestinal symptoms such as nausea and constipation were the least common. In an age- and gender-standardized comparison with a German normal population, all scales showed significant differences. Large differences were observed for role (27 points) and social functioning (27.8 points). Medium/ moderate differences in financial difficulties (15.7 points) and emotional functioning (14.2 points) were found (Figure 2). 

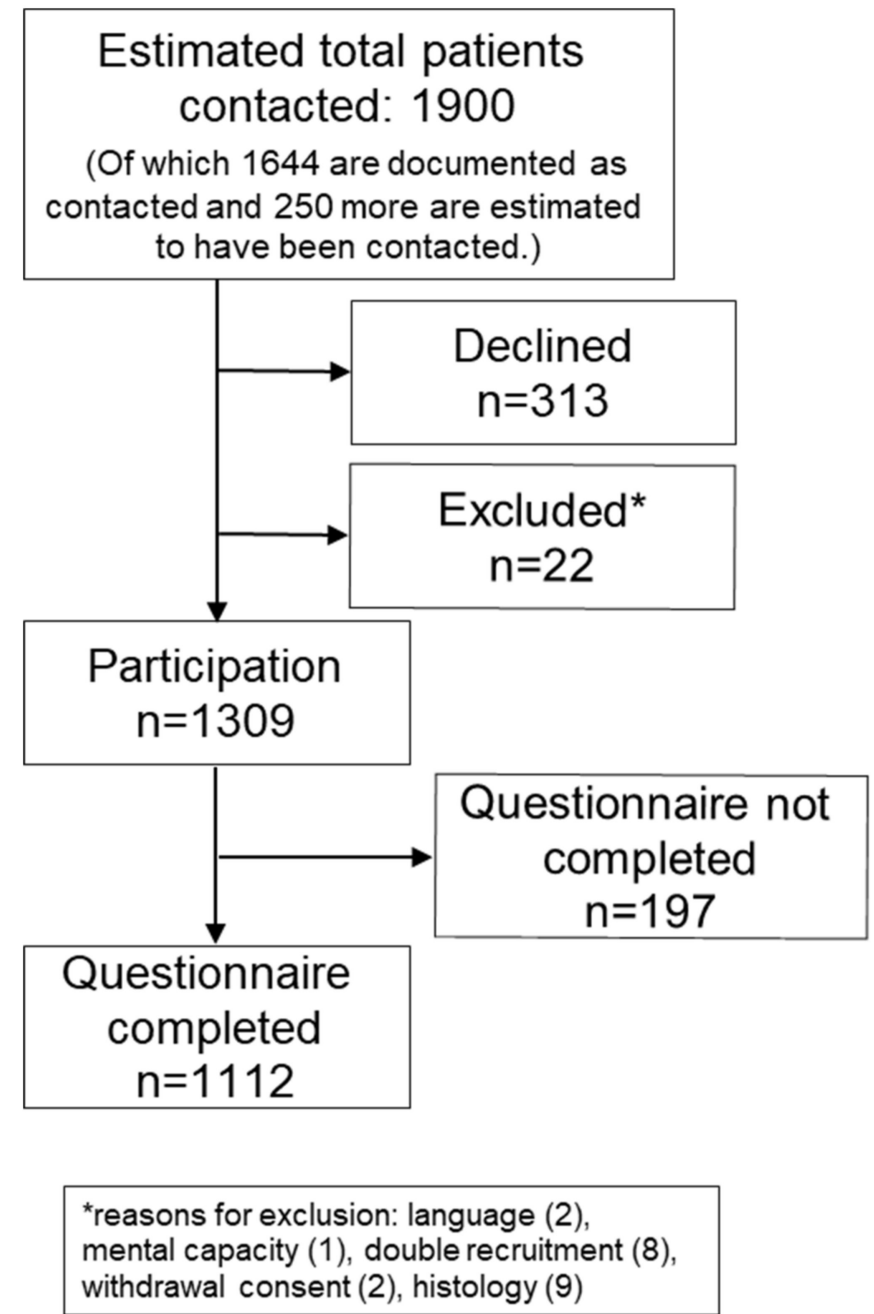

Figure 1. Study participation. Number of patients contacted overall had to be extrapolated from numbers of reporting study centers because not every study center documented contacted patients.

Table 1. Description of study population.

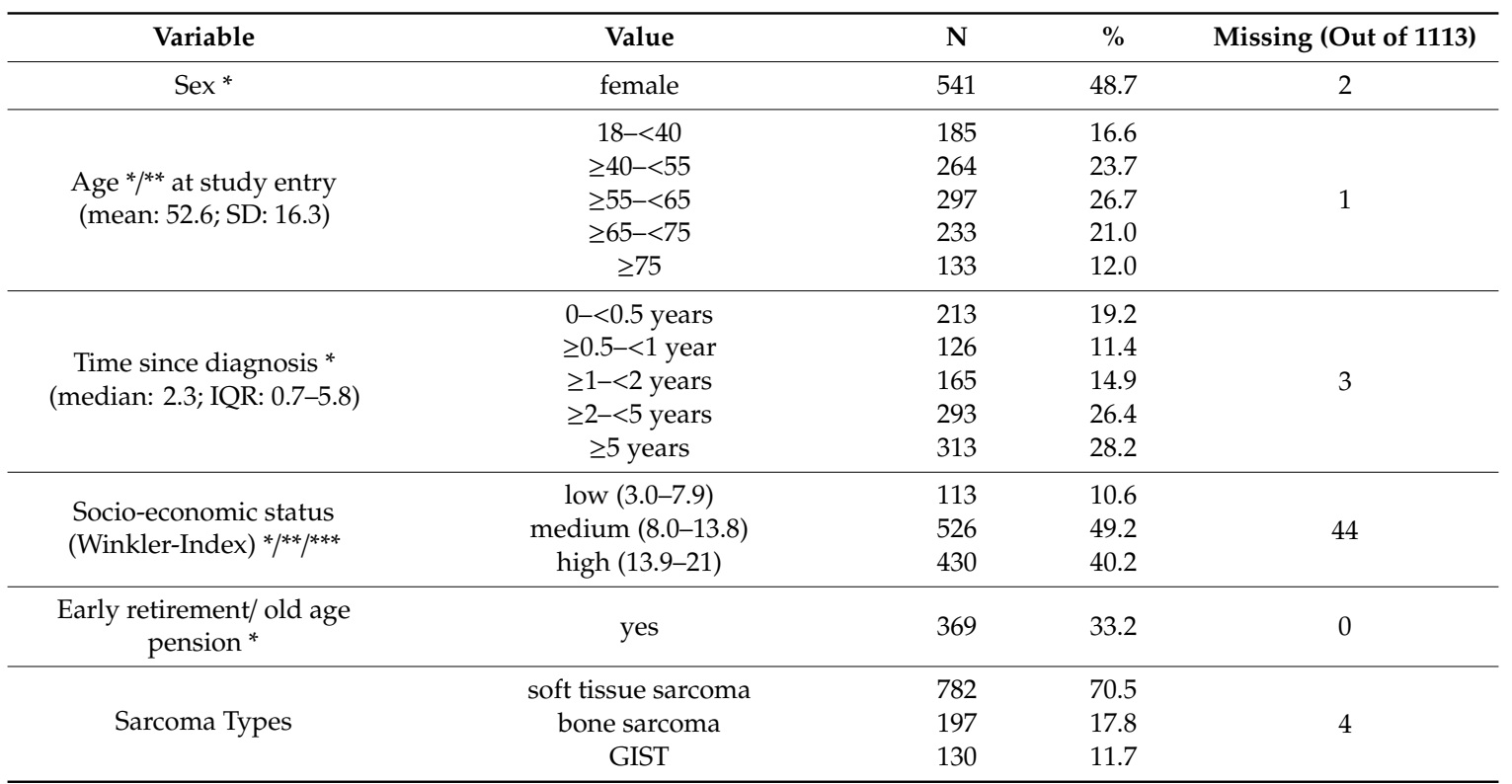


Table 1. Cont.

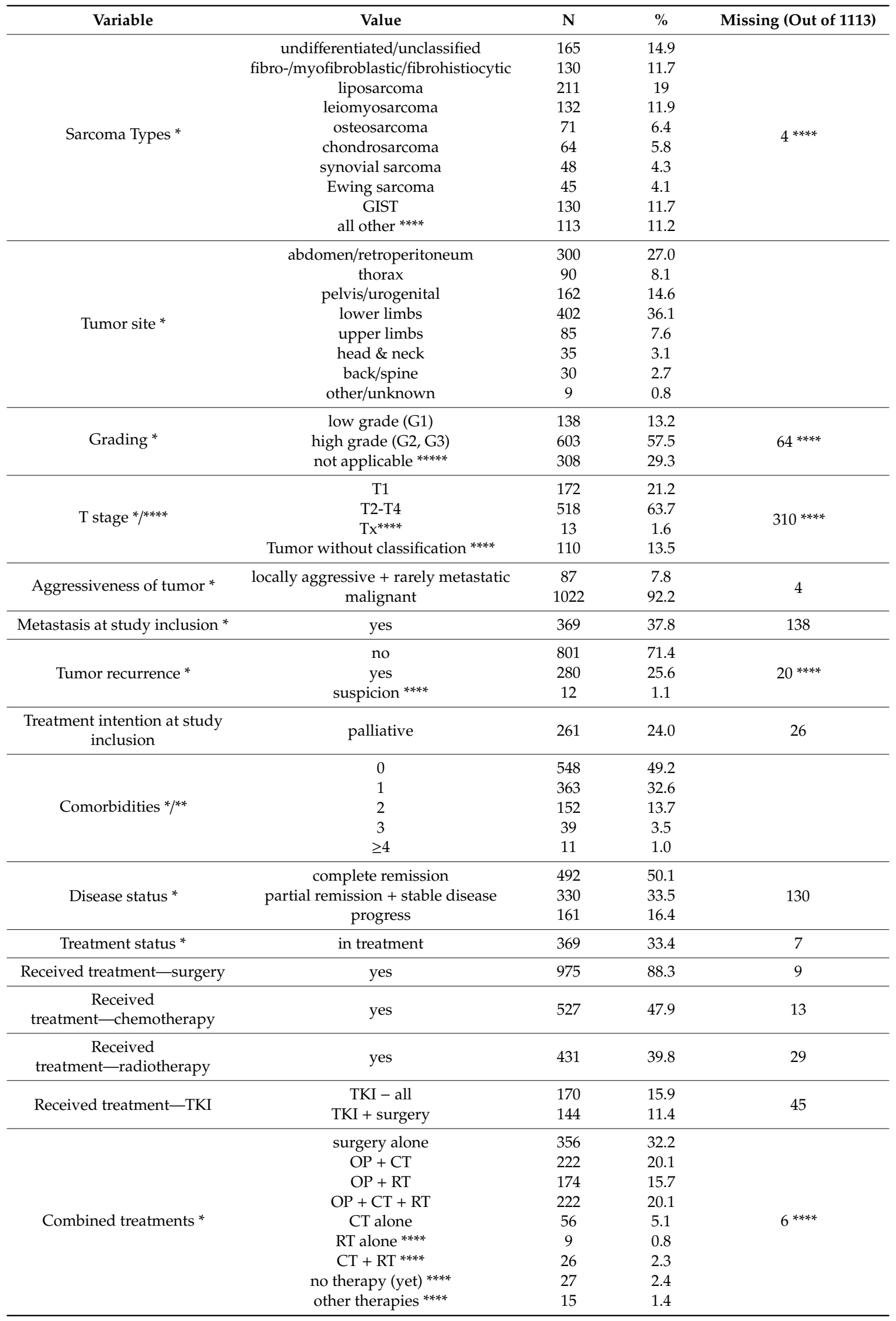

${ }^{*}$ variables in the model; ${ }^{* *}$ continuous model variable; ${ }^{* *} 35$ of these were later imputed. ${ }^{* * *}$ aggregated in the model. ***** tumor not graded, no surgery, neoadjuvant therapy. SD: standard deviation; IQR: inter-quartile range; OP: Surgery; TKI: Tyrosine kinase inhibitors; RT: radiotherapy; CT: chemotherapy. 

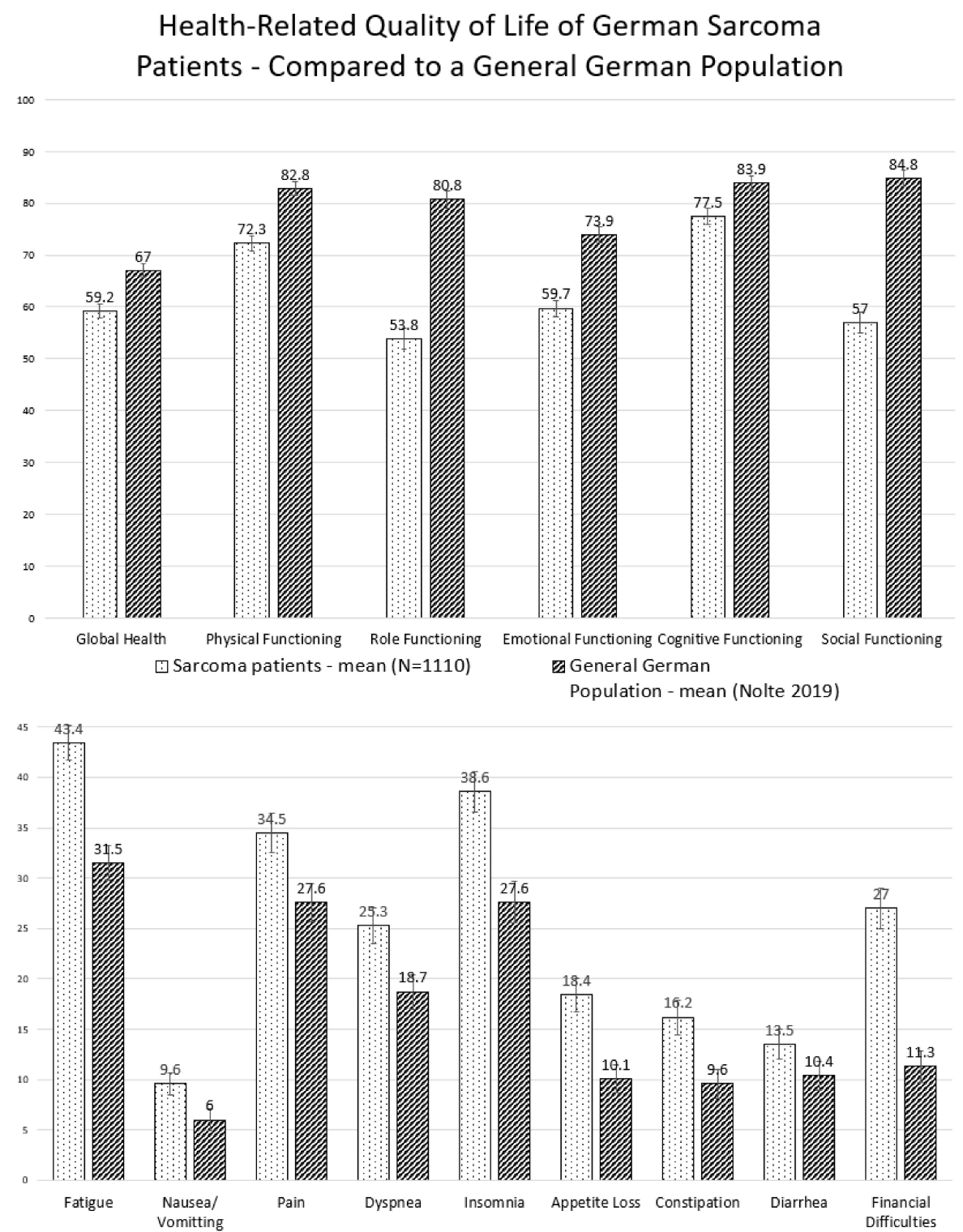

Figure 2. Age and gender standardized health-related quality of life of sarcoma patients and survivors. Mean values. Comparison with norm data from a German normal population (Nolte 2019). $\uparrow 95 \%$ confidence intervals. Large Differences: Role Functioning, Social Functioning; Medium/Moderate Differences: Financial Difficulties, Emotional Functioning; Small Differences: Fatigue, Dyspnea, Physical Functioning, Cognitive Functioning, Nausea/Vomiting, Pain, Insomnia, Appetite Loss, Diarrhea, Global Health, Constipation.

\subsection{Clinically Important Restrictions and Symptoms}

Between $39 \%$ and $63 \%$ of patients had clinically important limitations in the functioning scales. The highest percentages were emotional $(63 \%)$ and physical functioning $(60 \%)$. The proportion of clinically important symptoms varied between $14 \%$ and $56 \%$, with the lowest values for gastrointestinal symptoms such as constipation (14\%) and lack of appetite (16\%). The highest proportions were for pain $(56 \%)$, fatigue (51\%), and dyspnea (49\%) (Table 2$)$.

\subsection{Stratified Analyses}

Patients in palliative treatment showed significant differences compared to patients in curative situations in the majority of domains. Among the functional scales, the largest significant differences 
were found in social ( $57.9 \%$ vs. $42.3 \%)$, physical (70.8\% vs. $55.6 \%)$, and role function $(61.4 \%$ vs. $47.3 \%)$, and in symptom burden for dyspnea ( $63.7 \%$ vs. $44.6 \%)$, fatigue $(65.1 \%$ vs. $46.4 \%)$, and diarrhea ( $40.0 \%$ vs. 21.8 ). When comparing sarcoma types, bone sarcoma patients were more restricted than soft tissue sarcoma patients, with the greatest differences were in role function (61.9\% vs. $49.1 \%)$ and pain $(65.0 \%$ vs. $54.0 \%)$. GIST patients were generally less affected, but reported diarrhea more often than soft-tissue sarcoma patients ( $44.6 \%$ vs. $23.9 \%$ ) (Table 2$)$.

\subsection{Associated Factors in Multivariable Regression}

\subsubsection{Socio-Demographics}

Women showed significantly lower HRQoL values of trivial or small relevance than men in all eight domains. Higher age was significantly associated with worse values in five domains. Taking into account an age difference of 50 years, the differences in physical functioning were medium, in dyspnoea large. A higher Socioeconomic status (SES) was associated with better HRQoL in four domains. Comparing the lowest SES with the highest (3 vs. 21 points), the differences in pain are considered as large in physical functioning and as medium in general health. Patients in early retirement or collecting an old age pension had significantly better values in seven scales than those who were not retired The differences were medium in emotional functioning and small in the other domains (Table 3).

Table 2. Percentage of clinically important limitations and symptoms, stratified by treatment intention and sarcoma type.

\begin{tabular}{|c|c|c|c|c|c|c|c|c|c|c|c|c|}
\hline \multirow[t]{2}{*}{ Variable } & \multicolumn{2}{|c|}{$\begin{array}{c}\text { All Patients } \\
(\mathrm{N}=1003-1100)\end{array}$} & \multicolumn{2}{|c|}{$\begin{array}{c}\text { Curative } \\
(\mathrm{N}=818-825)\end{array}$} & \multicolumn{2}{|c|}{$\begin{array}{c}\text { Palliative } \\
(\mathrm{N}=258-261)\end{array}$} & \multicolumn{2}{|c|}{$\begin{array}{c}\text { Soft Tissue } \\
\text { Sarcoma } \\
(\mathrm{N}=775-780)\end{array}$} & \multicolumn{2}{|c|}{$\begin{array}{l}\text { Bone Sarcoma } \\
(N=195-197)\end{array}$} & \multicolumn{2}{|c|}{$\begin{array}{c}\text { GIST } \\
(\mathrm{N}=129-130)\end{array}$} \\
\hline & $\%$ & $95 \%$ CI & $\%$ & $95 \% \mathrm{CI}$ & $\%$ & $95 \% \mathrm{CI}$ & $\%$ & $95 \%$ CI & $\%$ & $95 \%$ CI & $\%$ & $95 \% \mathrm{CI}$ \\
\hline $\begin{array}{c}\text { Physical } \\
\text { Functioning }\end{array}$ & 59.5 & $\begin{array}{l}56.5 \\
62.4\end{array}$ & 55.6 & $\begin{array}{l}52.1 \\
59.0\end{array}$ & 70.8 & $\begin{array}{l}64.8 \\
76.2\end{array}$ & 58.8 & $\begin{array}{l}55.2 \\
62.3\end{array}$ & 67.0 & $\begin{array}{l}60.2 \\
73.5\end{array}$ & 50.8 & $\begin{array}{l}41.9 \\
59.6\end{array}$ \\
\hline $\begin{array}{c}\text { Role } \\
\text { Functioning }\end{array}$ & 50.7 & $\begin{array}{l}47.7 \\
53.7\end{array}$ & 47.3 & $\begin{array}{l}43.8 \\
50.7\end{array}$ & 61.4 & $\begin{array}{l}55.2 \\
67.4\end{array}$ & 49.1 & $\begin{array}{l}45.5 \\
52.7\end{array}$ & 61.9 & $\begin{array}{l}54.8 \\
68.7\end{array}$ & 42.6 & $\begin{array}{l}34.0 \\
51.6\end{array}$ \\
\hline $\begin{array}{c}\text { Emotional } \\
\text { Functioning }\end{array}$ & 62.7 & $\begin{array}{l}59.8 \\
65.6\end{array}$ & 61.2 & $\begin{array}{l}57.8 \\
64.6\end{array}$ & 67.7 & $\begin{array}{l}61.6 \\
73.3\end{array}$ & 63.2 & $\begin{array}{l}59.7 \\
66.6\end{array}$ & 61.4 & $\begin{array}{l}54.2 \\
68.3\end{array}$ & 61.5 & $\begin{array}{l}52.6 \\
70.0\end{array}$ \\
\hline $\begin{array}{l}\text { Cognitive } \\
\text { Functioning }\end{array}$ & 39.0 & $\begin{array}{l}36.1 \\
42.0\end{array}$ & 37.3 & $\begin{array}{l}34.0 \\
40.7\end{array}$ & 44.8 & $\begin{array}{l}38.7 \\
51.1\end{array}$ & 39.4 & $\begin{array}{l}35.9 \\
42.9\end{array}$ & 38.6 & $\begin{array}{l}31.8 \\
45.8\end{array}$ & 36.9 & $\begin{array}{l}28.6 \\
45.8\end{array}$ \\
\hline $\begin{array}{c}\text { Social } \\
\text { Functioning }\end{array}$ & 45.7 & $\begin{array}{l}42.8 \\
48.7\end{array}$ & 42.3 & $\begin{array}{l}38.9 \\
45.7\end{array}$ & 57.9 & $\begin{array}{l}51.6 \\
63.9\end{array}$ & 45.3 & $\begin{array}{l}41.8 \\
48.9\end{array}$ & 52.3 & $\begin{array}{l}45.1 \\
59.4\end{array}$ & 37.7 & $\begin{array}{l}29.4 \\
46.7\end{array}$ \\
\hline Fatigue & 50.9 & $\begin{array}{l}48.0 \\
53.9\end{array}$ & 46.4 & $\begin{array}{l}43.0 \\
49.9\end{array}$ & 65.1 & $\begin{array}{l}59.0 \\
70.9\end{array}$ & 51.2 & $\begin{array}{l}47.6 \\
54.7\end{array}$ & 53.3 & $\begin{array}{l}46.1 \\
60.4\end{array}$ & 45.0 & $\begin{array}{l}36.2 \\
54.0\end{array}$ \\
\hline Nausea/Vomiting & 27.5 & $\begin{array}{l}24.9 \\
30.2\end{array}$ & 23.4 & $\begin{array}{l}20.6 \\
26.5\end{array}$ & 40.6 & $\begin{array}{l}34.6 \\
46.8\end{array}$ & 25.4 & $\begin{array}{l}22.4 \\
28.6\end{array}$ & 29.9 & $\begin{array}{l}23.7 \\
36.9\end{array}$ & 35.4 & $\begin{array}{l}27.2 \\
44.3\end{array}$ \\
\hline Pain & 55.5 & $\begin{array}{l}52.6 \\
58.5\end{array}$ & 53.9 & $\begin{array}{l}50.4 \\
57.3\end{array}$ & 60.5 & $\begin{array}{l}54.3 \\
66.5\end{array}$ & 54.0 & $\begin{array}{l}50.4 \\
57.5\end{array}$ & 65.0 & $\begin{array}{l}57.9 \\
71.6\end{array}$ & 50.8 & $\begin{array}{l}41.9 \\
59.6\end{array}$ \\
\hline Dyspnea & 49.2 & $\begin{array}{l}46.2 \\
52.2\end{array}$ & 44.6 & $\begin{array}{l}41.2 \\
48.1\end{array}$ & 63.7 & $\begin{array}{l}57.3 \\
69.6\end{array}$ & 51.4 & $\begin{array}{l}47.8 \\
55.0\end{array}$ & 41.1 & $\begin{array}{l}34.2 \\
48.3\end{array}$ & 48.5 & $\begin{array}{l}39.6 \\
57.4\end{array}$ \\
\hline Insomnia & 35.3 & $\begin{array}{l}32.4 \\
38.2\end{array}$ & 35.4 & $\begin{array}{l}32.1 \\
38.8\end{array}$ & 36.8 & $\begin{array}{l}30.9 \\
43.0\end{array}$ & 34.7 & $\begin{array}{l}31.4 \\
38.2\end{array}$ & 33.5 & $\begin{array}{l}27.0 \\
40.6\end{array}$ & 41.1 & $\begin{array}{l}32.5 \\
50.1\end{array}$ \\
\hline Appetite Loss & 15.8 & $\begin{array}{l}13.7 \\
18.0\end{array}$ & 12.7 & $\begin{array}{l}10.5 \\
15.2\end{array}$ & 24.5 & $\begin{array}{l}19.4 \\
30.2\end{array}$ & 15.0 & $\begin{array}{l}12.6 \\
17.7\end{array}$ & 16.8 & $\begin{array}{l}11.8 \\
22.7\end{array}$ & 16.9 & $\begin{array}{l}10.9 \\
24.5\end{array}$ \\
\hline Constipation & 13.6 & $\begin{array}{l}11.6 \\
15.7\end{array}$ & 11.6 & $\begin{array}{c}9.5 \\
14.0\end{array}$ & 18.4 & $\begin{array}{l}13.9 \\
23.6\end{array}$ & 12.5 & $\begin{array}{l}10.2 \\
15.0\end{array}$ & 16.8 & $\begin{array}{l}11.8 \\
22.7\end{array}$ & 14.6 & $\begin{array}{c}9.0 \\
21.9\end{array}$ \\
\hline Diarrhea & 26.0 & $\begin{array}{l}23.5 \\
28.7\end{array}$ & 21.8 & $\begin{array}{l}19.0 \\
24.8\end{array}$ & 40.0 & $\begin{array}{l}34.0 \\
46.2\end{array}$ & 23.6 & $\begin{array}{l}20.6 \\
26.7\end{array}$ & 23.9 & $\begin{array}{l}18.1 \\
30.4\end{array}$ & 44.6 & $\begin{array}{l}35.9 \\
53.6\end{array}$ \\
\hline $\begin{array}{l}\text { Financial } \\
\text { Difficulties }\end{array}$ & 45.1 & $\begin{array}{l}42.2 \\
48.1\end{array}$ & 43.8 & $\begin{array}{l}40.4 \\
47.3\end{array}$ & 49.6 & $\begin{array}{l}43.4 \\
55.9\end{array}$ & 45.2 & $\begin{array}{l}41.6 \\
48.7\end{array}$ & 48.7 & $\begin{array}{l}41.5 \\
56.0\end{array}$ & 39.2 & $\begin{array}{l}30.8 \\
48.2\end{array}$ \\
\hline
\end{tabular}


Table 3. Results of the multivariable linear regression. \# no difference; \#\# small difference; \#\#\# moderate difference (Osoba 1998). * trivial difference; ** small difference; *** medium difference; ${ }^{* * * *}$ large difference (Cocks 2011); ${ }^{\text {II }} 35$ values imputed. For continuous variables we compared: Age: 50-year difference. SES: 18-point difference; Comorbidities: 4-point difference. Significant Differences: bold. $\mathrm{R}^{2}=$ coefficient of determination; B= non-standardized regression coefficient (indicating a B point increase or decrease in the respective QoL scale); $95 \%$ CI (lower;upper): 95\% confidence interval; $p=p$-value; na: not applicable; RT: radio therapy; CT: chemo therapy; SES: socioeconomic status.

\begin{tabular}{|c|c|c|c|c|c|c|c|c|c|c|c|c|c|}
\hline \multirow[t]{2}{*}{ Variable } & \multirow[t]{2}{*}{ Value } & \multicolumn{3}{|c|}{ General Health $\left(R^{2}=0.16\right)$} & \multicolumn{3}{|c|}{ Physical Functioning $\left(\mathrm{R}^{2}=0.19\right)$} & \multicolumn{3}{|c|}{ Social Functioning $\left(R^{2}=0.16\right)$} & \multicolumn{3}{|c|}{ Emotional Functioning $\left(R^{2}=0.08\right)$} \\
\hline & & B & $95 \%$ CI $(1 ; \mathbf{u})$ & $p$ & B & $95 \%$ CI $(1 ; \mathbf{u})$ & $p$ & B & $95 \%$ CI $(1 ; \mathrm{u})$ & $p$ & B & $95 \%$ CI $(1 ; \mathrm{u})$ & $p$ \\
\hline Sex & male vs. female & $-2.91 *$ & $-5.56 ;-0.27$ & 0.03 & $-4.77 *$ & $-7.53 ;-2.00$ & $<0.01$ & $-5.23 * *$ & $-9.07 ;-1.39$ & 0.01 & -6.62 \# & $-9.83 ;-3.41$ & $<0.01$ \\
\hline Age & increase per year & $-0.19 * *$ & $-0.33 ;-0.06$ & 0.01 & $-0.31^{* * *}$ & $-0.45 ;-0.17$ & $<0.01$ & -0.15 & $-0.34 ; 0.05$ & 0.14 & -0.08 & $-0.24 ; 0.08$ & 0.34 \\
\hline SES $\mathbb{I}$ & increase per point & $0.66 * * *$ & $0.31 ; 1.02$ & $<0.01$ & $0.92 * * *$ & $0.55 ; 1.29$ & $<0.01$ & 0.27 & $-0.24 ; 0.78$ & 0.30 & 0.39 & $-0.04 ; 0.82$ & 0.08 \\
\hline \multirow[t]{4}{*}{ Old age pension/early retirement } & $\begin{array}{l}\text { no vs. yes } \\
\text { liposarcoma }\end{array}$ & $\begin{array}{l}4.59 * * \\
\text { reference }\end{array}$ & $0.74 ; 8.43$ & 0.02 & $5.45^{* *}$ & $1.42 ; 9.47$ & 0.01 & $8.31^{* *}$ & $2.73 ; 13.88$ & $<0.01$ & 9.86 \#\#\# & $5.18 ; 14.54$ & $<0.01$ \\
\hline & undifferentiated/unclassified & -4.24 & $-9.18 ; 0.71$ & 0.09 & $-9.77 * *$ & $-14.95 ;-4.60$ & $<0.01$ & -6.69 & $-13.85 ; 0.48$ & 0.07 & -6.56 \# & $-12.56 ;-0.56$ & 0.03 \\
\hline & fibro-/myofibroblastic & -4.76 & $-9.98 ;-0.46$ & 0.07 & -3.04 & $-8.51 ; 2.42$ & 0.28 & -0.75 & $-9.32 ; 6.83$ & 0.85 & -3.42 & $-9.75 ; 2.92$ & 0.29 \\
\hline & GIST & 0.46 & $-6.41 ; 7.34$ & 0.90 & -0.62 & $-7.83 ; 6.59$ & 0.87 & 0.74 & $-9.24 ; 10.72$ & 0.88 & -0.07 & $-8.42 ; 8.28$ & 0.98 \\
\hline \multirow{9}{*}{ Sarcoma Type } & leiomyosarcoma & 0.07 & $-5.17 ; 5.31$ & 0.98 & -1.20 & $-6.60 ; 4.27$ & 0.67 & 2.21 & $-5.37 ; 9.78$ & 0.57 & -4.01 & $-10.34: 2.33$ & 0.22 \\
\hline & osteosarcoma & $-7.43 * *$ & $-14.50 ;-0.36$ & 0.04 & $-15.01 * * *$ & $-22.46 ;-7.68$ & $<0.01$ & -11.96 *** & $-22.21 ;-1.71$ & 0.02 & -9.75 \#\# & $-18.33 ;-1.18$ & 0.03 \\
\hline & synovial sarcoma & $-8.74 * *$ & $-16.09 ;-1.39$ & 0.02 & -7.67 & $-15.36 ; 0.23$ & 0.051 & -7.11 & $-17.78 ; 3.56$ & 0.19 & -3.40 & $-12.32 ; 5.52$ & 0.46 \\
\hline & Ewing sarcoma & $-13.24 * * *$ & $-21.50 ;-4.99$ & $<0.01$ & $-11.69 * *$ & $-20.33 ;-3.05$ & 0.01 & $-12.85^{* * *}$ & $-24.83 ;-0.87$ & 0.04 & -6.39 & $-16.41 ; 3.63$ & 0.21 \\
\hline & chondrosarcoma & -10.56 *** & $-17.19 ;-3.94$ & $<0.01$ & $-15.82 * * *$ & $-22.76 ;-8.89$ & $<0.01$ & $-18.8^{* * * *}$ & $-28.38 ;-9.15$ & $<0.01$ & -6.10 & $-14.14 ; 1.95$ & 0.14 \\
\hline & $\begin{array}{l}\text { all other } \\
\text { al her limbs }\end{array}$ & $\begin{array}{l}-0.56 \\
\text { reference }\end{array}$ & $-6.23 ; 4.95$ & 0.82 & -5.66 & $-11.54 ; 0.22$ & 0.06 & -5.06 & $-13.19 ; 3.08$ & 0.22 & -4.37 & $-11.17 ; 2.43$ & 0.21 \\
\hline & abdomen/retroperitoneum & 4.10 & $-0.28 ; 8.47$ & 0.07 & $5.83 * *$ & $1.26 ; 10.40$ & 0.01 & $7.17 * *$ & $0.83 ; 13.51$ & 0.03 & 0.13 & $-5.18 ; 5.43$ & 0.96 \\
\hline & thorax & 3.76 & $-1.45 ; 8.97$ & 0.16 & $8.38 * *$ & $2.90 ; 13.86$ & $<0.01$ & $8.94 * *$ & $1.37 ; 16.51$ & 0.02 & -2.38 & $-8.71 ; 3.96$ & 0.46 \\
\hline & pelvis/urogenital & 0.25 & $-4.00 ; 4.51$ & 0.91 & 1.59 & $-2.86 ; 6.04$ & 0.48 & -0.43 & $-6.60 ; 5.74$ & 0.89 & 0.31 & $-4.86 ; 5.48$ & 0.91 \\
\hline \multirow[t]{4}{*}{ Tumor Site } & upper limbs & $9.16 *$ & $3.91 ; 14.39$ & $<0.01$ & $12.54^{* *}$ & 7.07; 18.02 & $<0.01$ & $12.04 * * *$ & $4.74 ; 19.64$ & $<0.01$ & 1.99 & $-4.37 ; 8.35$ & 0.54 \\
\hline & head \& neck & 7.29 & $-0.38 ; 14.96$ & 0.06 & $10.78^{*}$ & $2.64 ; 18.81$ & 0.01 & $16.30^{* * * *}$ & $5.15 ; 27.44$ & $<0.01$ & 11.39 \#\# & $2.07 ; 20.70$ & 0.02 \\
\hline & back/spine & -1.17 & $-9.67 ; 7.33$ & 0.79 & 3.71 & $-5.18 ; 12.60$ & 0.41 & 0.27 & $-12.08 ; 12.61$ & 0.97 & 1.57 & $-8.76 ; 11.89$ & 0.77 \\
\hline & all other & 15.34 & $-4.14 ; 34.81$ & 0.12 & 6.86 & $-13.53 ; 27.25$ & 0.51 & 5.60 & $-22.70 ; 33.89$ & 0.70 & 3.04 & $-20.63 ; 26.71$ & 0.80 \\
\hline \multirow{2}{*}{ Grading } & $\mathrm{G} 1 \mathrm{G} 3$ & $\begin{array}{l}\text { reference } \\
-0.45\end{array}$ & $-4.87 ; 3.98$ & 0.84 & -3.44 & $-8.07 ; 1.18$ & 0.15 & $-6.84 * *$ & $-13.27 ;-0.43$ & 0.04 & -1.58 & $-6.94: 3.79$ & 0.57 \\
\hline & $\begin{array}{l}\text { other (unknown/na) } \\
\text { T1 }\end{array}$ & $\begin{array}{l}0.56 \\
\text { reference }\end{array}$ & $-4.91 ; 6.03$ & 0.84 & -1.10 & $-6.84 ; 4.64$ & 0.71 & -5.45 & $-13.40 ; 2.50$ & 0.18 & -3.82 & $-10.47 ; 2.83$ & 0.26 \\
\hline T-Stadium & $\begin{array}{c}\text { T2-T4 } \\
\text { T2 }\end{array}$ & 1.06 & $-3.07 ; 5.18$ & 0.62 & -2.54 & $\begin{array}{l}-6.84 ; 1.77 \\
-402.537\end{array}$ & 0.25 & -4.86 & $-10.85 ; 1.13$ & 0.11 & -2.54 & $-7.55 ; 2.47$ & 0.32 \\
\hline \multirow[t]{2}{*}{ Aggressiveness Tumor } & $\begin{array}{l}\text { other (unknown/na) } \\
\text { malignant vs. locally } \\
\text { aggressive + rarely metastatic }\end{array}$ & $\begin{array}{c}2.56 \\
2.96\end{array}$ & $\begin{array}{l}-1.95 ; 7.06 \\
-4.06 ; 9.99\end{array}$ & $\begin{array}{l}0.27 \\
0.41\end{array}$ & $\begin{array}{c}0.68 \\
-2.16\end{array}$ & $\begin{array}{l}-4.02 ; 5.37 \\
-9.51 ; 5.18\end{array}$ & $\begin{array}{l}0.78 \\
0.56\end{array}$ & $\begin{array}{l}-2.93 \\
-4.34\end{array}$ & $\begin{array}{c}-9.46 ; 3.60 \\
-14.54 ; 5.86\end{array}$ & $\begin{array}{l}0.40 \\
0.40\end{array}$ & $\begin{array}{l}-0.75 \\
-3.24\end{array}$ & $\begin{array}{l}-6.21 ; 4.72 \\
-11.78 ; 5.29\end{array}$ & $\begin{array}{l}0.79 \\
0.46\end{array}$ \\
\hline & $\begin{array}{l}\text { no } \\
\text { ves }\end{array}$ & $\begin{array}{l}\text { reference } \\
-1.86\end{array}$ & & & & $-6.85 ; 0.70$ & 0.11 & -3.83 & $-9.06 ; 1.41$ & 0.15 & -1.70 & $-6.08 ; 2.68$ & 0.45 \\
\hline Metastasis till study inclusion & $\begin{array}{l}\text { yes } \\
\text { unknown }\end{array}$ & $\begin{array}{l}-1.00 \\
-1.82\end{array}$ & $-6.18 ; 2.54$ & 0.41 & $\begin{array}{l}-3.07 \\
-2.14\end{array}$ & $\begin{array}{l}-0.03,69 ; 2.41 \\
-6.69\end{array}$ & 0.36 & 0.86 & $\begin{array}{l}-3,00,1.41 \\
-5.47 ; 7.20\end{array}$ & 0.79 & $\begin{array}{l}-1.10 \\
-2.17\end{array}$ & $\begin{array}{l}-0.00,2.00 \\
-7.47 ; 3.13\end{array}$ & 0.42 \\
\hline Comorbidities & $\begin{array}{l}\text { increase per comorbidity } \\
\text { complete remission }\end{array}$ & $\begin{array}{l}-2.78 * * * \\
\text { reference }\end{array}$ & $-4.43 ;-1.12$ & $<0.01$ & $-3.43^{* *}$ & $-5.16 ;-1.69$ & $<0.01$ & $-3.41^{* * *}$ & $-5.81 ;-1.00$ & 0.01 & -1.90 & $-3.91 ; 0.07$ & 0.07 \\
\hline \multirow{3}{*}{ Disease status } & part. remission + stable disease & -3.17 & $-6.77 ; 0.43$ & 0.08 & -0.14 & $-3.91 ; 3.63$ & 0.94 & $-5.51 * *$ & $-10.74 ;-0.28$ & 0.04 & -4.11 & $-8.48 ; 0.27$ & 0.07 \\
\hline & & $-7.98 * *$ & $-12.87 ;-3.09$ & $<0.01$ & -2.24 & $-7.34 ; 2.86$ & 0.39 & -6.95 & $-14.03 ; 0.13$ & 0.054 & -5.32 & $-11.25 ; 0.61$ & 0.08 \\
\hline & unknown & -0.39 & $-5.46 ; 4.69$ & 0.88 & 0.68 & $-4.63 ; 5.99$ & 0.80 & $-8.54^{* *}$ & $-15.91 ;-1.17$ & 0.02 & -4.25 & $-10.42 ; 1.91$ & 0.18 \\
\hline Treatment status & no vs. yes & $-6.32 * *$ & $-9.90 ;-2.75$ & $<0.01$ & $-5.03^{* *}$ & $-8.76 ;-1.30$ & 0.01 & $-5.90^{* *}$ & $-11.08 ;-0.71$ & 0.03 & -0.67 & $-5.01 ; 3.66$ & 0.76 \\
\hline
\end{tabular}


Table 3. Cont

\begin{tabular}{|c|c|c|c|c|c|c|c|c|c|c|c|c|c|}
\hline \multirow[t]{2}{*}{ Variable } & \multirow[t]{2}{*}{ Value } & \multicolumn{3}{|c|}{ General Health $\left(R^{2}=0.16\right)$} & \multicolumn{3}{|c|}{ Physical Functioning $\left(\mathrm{R}^{2}=0.19\right)$} & \multicolumn{3}{|c|}{ Social Functioning $\left(R^{2}=0.16\right)$} & \multicolumn{3}{|c|}{ Emotional Functioning $\left(R^{2}=0.08\right)$} \\
\hline & & B & $95 \%$ CI $(1 ; \mathrm{u})$ & $p$ & B & $95 \%$ CI $(1 ; u)$ & $p$ & B & $95 \%$ CI $(1 ; \mathbf{u})$ & $p$ & B & $95 \%$ CI $(1 ; u)$ & $p$ \\
\hline \multirow{7}{*}{ Combined Treatments } & surgery alone & reference & & & & & & & & & & & \\
\hline & surgery $+\mathrm{CT}$ & -0.39 & $-4.65 ; 3.88$ & 0.86 & $-4.63^{* *}$ & $-9.10 ;-0.16$ & 0.04 & -4.64 & $-10.84 ; 1.56$ & 0.14 & 2.72 & $-2.46 ; 7.91$ & 0.30 \\
\hline & surgery + RT & 0.02 & $-4.32 ; 4.37$ & 0.99 & -2.35 & $-6.89 ; 2.20$ & 0.31 & -4.71 & $-11.02 ; 1.61$ & 0.14 & -0.40 & $-5.68 ; 4.88$ & 0.88 \\
\hline & surgery + CT + RT & -1.17 & $-5.61 ; 3.27$ & 0.61 & $-5.25^{* *}$ & $-9.89 ;-0.61$ & 0.03 & -4.95 & $-11.39 ; 1.49$ & 0.13 & -2.72 & $-8.10 ; 2.67$ & 0.32 \\
\hline & CT alone & -0.56 & $-6.57 ; 7.68$ & 0.88 & -6.94 & $-14.43 ; 0.55$ & 0.07 & -5.78 & $-16.13 ; 4.57$ & 0.27 & 7.40 & $-1.32 ; 16.12$ & 0.10 \\
\hline & all other & -1.81 & $-7.66 ; 4.04$ & 0.54 & -3.76 & $-9.86 ; 2.34$ & 0.23 & -4.31 & $-12.78 ; 4.16$ & 0.32 & -2.31 & $-9.40 ; 4.77$ & 0.52 \\
\hline & $0-<6$ months & reference & & & & & & & & & & & \\
\hline \multirow{4}{*}{ Time since diagnosis } & $6-<12$ months & 0.92 & $-4.26 ; 6.10$ & 0.73 & -2.89 & $-8.32 ; 2.54$ & 0.30 & -3.18 & $-10.70 ; 4.35$ & 0.41 & -0.08 & $-6.37 ; 6.22$ & 0.98 \\
\hline & $12-<24$ months & $6.68^{* *}$ & $1.64 ; 11.72$ & 0.01 & -1.67 & $-6.93 ; 3.60$ & 0.54 & 3.91 & $-3.41 ; 11.22$ & 0.30 & 2.52 & $-3.62 ; 8.66$ & 0.42 \\
\hline & $24-<60$ months & $8.67^{* *}$ & $3.89 ; 13.44$ & $<0.01$ & 0.91 & $-4.07 ; 5.90$ & 0.72 & 6.25 & $-0.68 ; 13.18$ & 0.08 & 2.69 & $-3.11 ; 8.49$ & 0.36 \\
\hline & $\begin{array}{l}60 \text { months or more } \\
\text { no }\end{array}$ & $\begin{array}{l}7.73^{* *} \\
\text { reference }\end{array}$ & $2.60 ; 12.87$ & $<0.01$ & -1.88 & $-7.24 ; 3.49$ & 0.49 & $9.54 * *$ & $2.09 ; 16.99$ & 0.01 & $7.15^{\# \#}$ & $0.91 ; 13.39$ & 0.03 \\
\hline \multirow{2}{*}{ Tumor recurrence } & yes & $-4.96^{*}$ & $-8.43 ;-1.49$ & 0.01 & $-3.67 *$ & $-7.30 ;-0.04$ & 0.04 & $-8.35^{* *}$ & $-13.38 ;-3.32$ & $<0.01$ & $-5.52 \ldots$ & $-9.73 ;-1.31$ & 0.01 \\
\hline & unknown & 0.73 & $-7.75 ; 9.21$ & 0.87 & -2.80 & $-11.67 ; 6.09$ & 0.54 & 2.27 & $-10.05 ; 14.59$ & 0.72 & -1.26 & $-11.75 ; 9.23$ & 0.81 \\
\hline \multirow[t]{2}{*}{ Variable } & Value & \multicolumn{3}{|c|}{ Role Functioning $\left(R^{2}=0.16\right)$} & \multicolumn{3}{|c|}{ Pain $\left(R^{2}=0.10\right)$} & \multicolumn{3}{|c|}{ Fatigue $\left(R^{2}=0.16\right)$} & \multicolumn{3}{|c|}{ Dyspnea $\left(R^{2}=0.14\right)$} \\
\hline & & B & $95 \%$ CI $(1 ; \mathbf{u})$ & $\mathrm{p}$ & B & $95 \%$ CI $(1 ; \mathrm{u})$ & $\mathrm{p}$ & B & $95 \%$ CI $(1 ; \mathrm{u})$ & $\mathrm{p}$ & B & $95 \%$ CI $(1 ; \mathbf{u})$ & $\mathrm{p}$ \\
\hline Sex & male vs. female & $-6.11 * *$ & $-10.01 ;-2.22$ & $<0.01$ & $4.16^{*}$ & $0.36 ; 7.97$ & 0.03 & 8.45 ** & $5.13 ; 11.76$ & $<0.01$ & 5.50 ** & $1.95 ; 9.05$ & $<0.01$ \\
\hline Age & increase per year & -0.20 ** & $-0.40 ;-0.004$ & 0.045 & 0.12 & $-0.07 ; 0.31$ & 0.22 & $0.26^{* * *}$ & $0.10 ; 0.43$ & $<0.01$ & $0.33^{* * * * *}$ & $0.15 ; 0.51$ & $<0.01$ \\
\hline SESII & increase per point & 0.45 & $-0.07 ; 0.97$ & 0.09 & $-1.25 * * * *$ & $-1.76 ;-0.74$ & $<0.01$ & $-0.59 * *$ & $-1.03 ;-0.15$ & 0.01 & -0.23 & $-0.71 ; 0.24$ & 0.33 \\
\hline \multirow[t]{4}{*}{ Old age pension/early retirement } & $\begin{array}{l}\text { no vs. yes } \\
\text { liposarcoma }\end{array}$ & $\begin{array}{l}8.18 * * \\
\text { reference }\end{array}$ & $2.51 ; 13.85$ & 0.01 & -2.96 & $-8.49 ; 2.53$ & 0.29 & -8.82 ** & $-13.64 ;-4.00$ & $<0.01$ & $-6.78^{* *}$ & $-11.98 ;-1.62$ & 0.01 \\
\hline & undifferentiated/unclassified & $-9.74 * *$ & $-17.02 ;-2.47$ & 0.01 & 5.14 & $-1.95 ; 12.38$ & 0.16 & 4.54 & $-1.64 ; 10.73$ & 0.15 & 1.13 & $-5.52 ; 7.77$ & 0.74 \\
\hline & fibro-/myofibroblastic & -5.62 & $-13.31 ; 2.07$ & 0.15 & 4.68 & $-2.83 ; 12.19$ & 0.22 & 3.25 & $-3.29 ; 9.79$ & 0.33 & -0.53 & $-7.55 ; 6.49$ & 0.88 \\
\hline & GIST & 6.88 & $-3.26 ; 17.02$ & 0.18 & 5.71 & $-4.18 ; 15.61$ & 0.26 & 0.30 & $-8.33 ; 8.83$ & 0.95 & $-9.76 * * *$ & $-18.98 ;-0.54$ & 0.04 \\
\hline \multirow{6}{*}{ Sarcoma Type } & leiomyosarcoma & 1.95 & $-5.75 ; 9.63$ & 0.62 & -2.40 & $-9.92 ; 5.12$ & 0.53 & 0.08 & $-6.46 ; 6.62$ & 0.98 & -2.56 & $-9.58 ; 4.46$ & 0.48 \\
\hline & osteosarcoma & $-13.79 * *$ & $-24.21 ;-3.38$ & 0.01 & 8.94 & $-1.22 ; 19.11$ & 0.09 & $10.25^{* *}$ & $1.40 ; 19.10$ & 0.02 & 5.10 & $-4.37 ; 14.58$ & 0.29 \\
\hline & synovial sarcoma & -8.72 & $-19.55 ; 2.12$ & 0.12 & 4.69 & $-5.89 ; 15.27$ & 0.38 & 5.05 & $-4.17 ; 14.26$ & 0.28 & 7.45 & $-2.40 ; 17.23$ & 0.14 \\
\hline & Ewing sarcoma & -13.50 ** & $-25.67 ;-1.33$ & 0.03 & 8.67 & $-3.21 ; 20.55$ & 0.15 & 11.94 ** & $1.60 ; 22.29$ & 0.02 & 2.93 & $-8.13 ; 13.99$ & 0.60 \\
\hline & chondrosarcoma & $-19.55^{* * *}$ & $-29.31 ;-9.78$ & $<0.01$ & $14.78^{* * * *}$ & $5.24 ; 24.31$ & $<0.01$ & $9.09 * *$ & $0.79 ; 17.34$ & 0.03 & 1.43 & $-7.45 ; 10.32$ & 0.75 \\
\hline & $\begin{array}{c}\text { all other } \\
\text { lower limbs }\end{array}$ & $\begin{array}{l}-4.85 \\
\text { reference }\end{array}$ & $-13.11 ; 3.41$ & 0.25 & 2.78 & $-5.29 ; 10.84$ & 0.50 & 5.26 & $-1.76 ; 12.28$ & 0.14 & -0.40 & $-7.92 ; 7.11$ & 0.92 \\
\hline \multirow[t]{7}{*}{ Tumor Site } & abdomen/retroperitoneum & $9.12 * *$ & $2.68 ; 15.57$ & 0.01 & -8.81 ** & $-15.1 ;-2.52$ & 0.01 & -0.90 & $-6.28 ; 4.58$ & 0.75 & 4.11 & $-1.76 ; 9.99$ & 0.17 \\
\hline & thorax & 9.53 * & $1.87 ; 17.25$ & 0.02 & $-12.21 * *$ & $-19.7 ;-4.71$ & $<0.01$ & -1.33 & $-7.89 ; 5.21$ & 0.69 & $8.33^{* * *}$ & $1.34 ; 15.31$ & 0.02 \\
\hline & pelvis/urogenital & $7.10^{* *}$ & $0.83 ; 13.37$ & 0.03 & -2.99 & $-9.11 ; 3.14$ & 0.34 & 1.05 & $-4.28 ; 6.38$ & 0.70 & -2.12 & $-7.84 ; 3.59$ & 0.47 \\
\hline & upper limbs & $10.47^{* *}$ & $2.75 ; 18.19$ & 0.01 & $-14.28 * * *$ & $-21.8 ;-6.74$ & $<0.01$ & $-7.41^{* *}$ & $-13.98 ;-0.85$ & 0.03 & -1.55 & $-8.56 ; 5.46$ & 0.67 \\
\hline & head \& neck & 13.45 ** & $2.14 ; 24.77$ & 0.02 & $-14.03^{* * *}$ & $-25.0 ;-2.98$ & 0.01 & -6.25 & $-15.89 ; 3.38$ & 0.20 & -4.45 & $-14.87 ; 5.98$ & 0.40 \\
\hline & back/spine & -2.55 & $-15.09 ; 9.99$ & 0.69 & 1.65 & $-10.59 ; 13.9$ & 0.79 & 4.13 & $-6.53 ; 14.79$ & 0.45 & 6.17 & $-5.21 ; 17.56$ & 0.29 \\
\hline & $\begin{array}{l}\text { all other } \\
\text { G1 }\end{array}$ & $\begin{array}{l}9.13 \\
\text { reference }\end{array}$ & $-19.62 ; 37.87$ & 0.53 & -25.55 & $-53.60 ; 2.51$ & 0.07 & 0.27 & $-24.17 ; 24.71$ & 0.98 & -7.45 & $-33.54 ; 18.63$ & 0.58 \\
\hline \multirow{2}{*}{ Grading } & G2/G3 & -4.71 & $-11.23 ; 1.81$ & 0.16 & 2.82 & $-3.55 ; 9.19$ & 0.39 & 3.83 & $-1.72 ; 9.37$ & 0.18 & 0.26 & $-5.70 ; 6.21$ & 0.93 \\
\hline & $\begin{array}{l}\text { other (unknown/na) } \\
\mathrm{T} 1\end{array}$ & $\begin{array}{l}-5.44 \\
\text { reference }\end{array}$ & $-13.52 ; 2.54$ & 0.19 & 1.88 & $-6.00 ; 9.76$ & 0.64 & 3.36 & $-3.51 ; 10.22$ & 0.34 & 3.41 & $-3.95 ; 10.77$ & 0.36 \\
\hline \multirow[t]{2}{*}{ T-Stadium } & T2-T4 & -1.91 & $-7.98 ; 4.16$ & 0.54 & 0.28 & $-5.65 ; 6.20$ & 0.93 & -2.17 & $-7.34 ; 3.00$ & 0.41 & -2.47 & $-8.02 ; 3.08$ & 0.38 \\
\hline & other (unknown/na) & 2.61 & $-4.01 ; 9.23$ & 0.44 & 1.00 & $-5.37 ; 7.57$ & 0.74 & $-6.27^{* *}$ & $-11.91 ;-0.63$ & 0.03 & -6.72 ** & $-12.79 ;-0.66$ & 0.03 \\
\hline \multirow[t]{2}{*}{ Aggressiveness Tumor } & $\begin{array}{l}\text { malignant vs. locally } \\
\text { aggressive + rarely metastatic }\end{array}$ & 1.14 & $-9.21 ; 11.50$ & 0.83 & 8.44 & $-1.67 ; 18.54$ & 0.10 & -2.80 & $-11.61 ; 6.01$ & 0.53 & -3.71 & $-13.17 ; 5.74$ & 0.44 \\
\hline & no & reference & & & & & & & & & & & \\
\hline Metastasis till study inclusion & yes & -5.07 & $-10.38 ; 0.25$ & 0.06 & -0.11 & $-5.30 ; 5.08$ & 0.97 & 4.49 & $-0.03 ; 9.02$ & 0.052 & $7.81^{* *}$ & $2.96 ; 12.67$ & $<0.01$ \\
\hline & unknown & -2.19 & $-8.60 ; 4.22$ & 0.50 & 1.64 & $-4.62 ; 7.89$ & 0.61 & 2.92 & $-2.55 ; 8.39$ & 0.30 & 2.31 & $-3.55 ; 8.17$ & 0.44 \\
\hline
\end{tabular}


Table 3. Cont.

\begin{tabular}{|c|c|c|c|c|c|c|c|c|c|c|c|c|c|}
\hline \multirow[t]{2}{*}{ Variable } & \multirow[t]{2}{*}{ Value } & \multicolumn{3}{|c|}{ General Health $\left(R^{2}=0.16\right)$} & \multicolumn{3}{|c|}{ Physical Functioning $\left(R^{2}=0.19\right)$} & \multicolumn{3}{|c|}{ Social Functioning $\left(R^{2}=0.16\right)$} & \multicolumn{3}{|c|}{ Emotional Functioning $\left(R^{2}=0.08\right)$} \\
\hline & & B & $95 \%$ CI $(1 ; \mathrm{u})$ & $p$ & B & $95 \%$ CI $(1 ; u)$ & $p$ & B & $95 \%$ CI $(1 ; u)$ & $p$ & B & $95 \%$ CI $(1 ; \mathrm{u})$ & $p$ \\
\hline Comorbidities & $\begin{array}{l}\text { increase per comorbidity } \\
\text { complete remission }\end{array}$ & $\begin{array}{l}-3.98 * * \\
\text { reference }\end{array}$ & $-6.42 ;-1.54$ & $<0.01$ & $2.69 * *$ & $0.30 ; 5.07$ & 0.03 & $4.11^{* * *}$ & $2.03 ; 6.19$ & $<0.01$ & $4.44^{* * * * *}$ & $2.23 ; 6.66$ & $<0.01$ \\
\hline \multirow{3}{*}{ Disease status } & part. remission + stable disease & -3.95 & $-9.26 ; 1.37$ & 0.15 & 4.63 & $-0.56 ; 9.82$ & 0.08 & $5.13 * *$ & $0.61 ; 9.61$ & 0.03 & 3.07 & $-1.77 ; 7.92$ & 0.21 \\
\hline & progress & -5.65 & $-12.84 ; 1.55$ & 0.12 & 3.75 & $-3.27 ; 10.77$ & 0.30 & 2.96 & $-3.16 ; 9.08$ & 0.34 & 6.06 & $-0.49 ; 12.61$ & 0.07 \\
\hline & unknown & -4.51 & $-11.99 ; 2.97$ & 0.23 & 3.17 & $-4.13: 10.43$ & 0.40 & 3.05 & $-3.32 ; 9.41$ & 0.35 & 4.71 & $-2.09 ; 11.51$ & 0.17 \\
\hline \multirow[t]{2}{*}{ Treatment status } & $\begin{array}{l}\text { no vs. yes } \\
\text { surgery alone }\end{array}$ & $\begin{array}{l}-11.86 * * \\
\text { reference }\end{array}$ & $-17.12 ; 6.60$ & $<0.01$ & 4.34 & $-0.80 ; 9.48$ & 0.10 & $7.46^{* *}$ & $2.98 ; 11.93$ & 0.001 & 6.63 ** & $1.85 ; 11.41$ & 0.01 \\
\hline & surgery $+\mathrm{CT}$ & -1.18 & $-7.48 ; 5.13$ & 0.71 & 1.09 & $-5.06 ; 7.24$ & 0.73 & 3.39 & $-1.94 ; 8.75$ & 0.21 & 4.53 & $-1.20 ; 10.26$ & 0.12 \\
\hline \multirow{4}{*}{ Combined Treatments } & surgery + RT & -2.79 & $-9.20 ; 3.61$ & 0.39 & 5.74 & $-0.52 ; 12.00$ & 0.07 & 4.12 & $-1.33 ; 9.57$ & 0.14 & 4.32 & $-1.53 ; 10.17$ & 0.15 \\
\hline & surgery $+\mathrm{CT}+\mathrm{RT}$ & -2.64 & $-9.18 ; 3.90$ & 0.43 & 4.36 & $-2.03 ; 10.75$ & 0.18 & $7.93^{* *}$ & $2.36 ; 13.49$ & 0.01 & 1.29 & $-4.66 ; 7.24$ & 0.67 \\
\hline & CT alone & -0.71 & $-11.22 ; 9.80$ & 0.89 & 4.28 & $-5.99 ; 14.56$ & 0.41 & 10.28 ** & $1.34 ; 19.22$ & 0.02 & 4.07 & $-5.56 ; 13.70$ & 0.41 \\
\hline & all other & 2.18 & $-6.43 ; 10.78$ & 0.62 & 10.57 ** & $2.16 ; 18.98$ & 0.01 & 5.50 & $-1.82 ; 12.82$ & 0.14 & -0.96 & $-8.78 ; 6.87$ & 0.81 \\
\hline \multirow[t]{5}{*}{ Time since diagnosis } & $0-<6$ months & reference & & & & & & & & & & & \\
\hline & $6-<12$ months & -2.20 & $-9.86 ; 5.45$ & 0.57 & 5.27 & $-2.20 ; 12.74$ & 0.17 & 3.68 & $-2.82 ; 10.18$ & 0.27 & 1.31 & $-5.66 ; 8.27$ & 0.71 \\
\hline & $12-<24$ months & 1.30 & $-6.12 ; 8.73$ & 0.73 & 0.66 & $-6.60 ; 7.91$ & 0.86 & 4.16 & $-2.16 ; 10.48$ & 0.20 & $7.51 * *$ & $0.73 ; 14.31$ & 0.03 \\
\hline & $24-<60$ months & 6.76 & $-0.26 ; 13.79$ & 0.06 & 2.56 & $-4.31 ; 9.43$ & 0.47 & 1.74 & $-4.25 ; 7.72$ & 0.57 & 3.21 & $-3.20 ; 9.62$ & 0.33 \\
\hline & 60 months or more & 4.84 & $-2.72 ; 12.40$ & 0.21 & 0.13 & $-7.26 ; 7.53$ & 0.97 & 0.80 & $-5.63 ; 7.24$ & 0.81 & 4.80 & $-2.10 ; 11.70$ & 0.17 \\
\hline \multirow{2}{*}{ Tumor recurrence } & $\begin{array}{l}\text { no } \\
\text { yes }\end{array}$ & $\begin{array}{l}\text { reference } \\
-6.41^{* *}\end{array}$ & $-11.53 ; 1.30$ & 0.01 & $6.31 * *$ & $1.32 ; 11.30$ & 0.01 & $5.17 * *$ & $0.82 ; 9.52$ & 0.02 & 0.87 & $-3.78 ; 5.52$ & 0.71 \\
\hline & unknown & $\begin{array}{l}-0.41 \\
1.12\end{array}$ & $-11.40 ; 13.63$ & 0.86 & -1.60 & $-13.81 ; 10.6$ & 0.80 & -2.35 & $-12.99 ; 8.24$ & 0.67 & 9.35 & $-2.21 ; 20.91$ & 0.11 \\
\hline
\end{tabular}




\subsubsection{Tumor Sites}

In many of the evaluated tumor sites, we found a variety of significant and relevant differences. With lower limbs as the reference, patients with sarcomas of the upper limbs had better HRQoL outcomes of small and medium relevance in six scales. Patients with tumors of the head and neck scored better in five domains, in emotional functioning and pain those differences are considered as medium. Patients with abdominal or retroperitoneal sarcomas as well as thoracic sarcomas scored better in four domains. The latter performed worse in dyspnea. Patients with sarcomas of the pelvis reached a better outcome in role functioning. The differences were mainly found in physical, social, and role functioning and in pain symptoms (Table 3).

\subsubsection{Sarcoma Types}

The comparison of the main nine sarcoma subtypes with liposarcoma as the most common as the reference showed that patients with one of the three main bone sarcoma types had the worst HRQoL outcomes. Patients with osteosarcoma and chondrosarcoma performed worse in six scales, those with Ewing sarcoma in five. The social functioning difference for chondrosarcoma patients is considered large. Differences between types of soft tissue sarcomas were observed as well. Patients with undifferentiated/ unclassified sarcomas scored worse in three scales, while those with synovial sarcomas had a poorer general health score. GIST patients had a better outcome in dyspnea. The differences were mainly found in general health, physical, social, and role functioning and in fatigue. No significant differences were found comparing patients with locally aggressive/rarely metastatic tumors with malignant ones (Table 3).

\subsubsection{Tumor-Related Factors}

With low grade tumors as a reference, patients with high-grade tumors had lower HRQoL scores of small relevance in social functioning, while those with larger tumors showed no significant differences compared to those with smaller tumors. Metastases in the course of the disease had a negative effect on dyspnea. Patients who suffered from a recurrence of the tumor reported worse outcomes in seven domains. The differences here were trivial or small (Table 3).

\subsubsection{Disease and Treatment Status}

The presence and number of comorbidities were associated with poorer HRQoL values in seven domains. Patients with stable disease or in partial remission had worse HRQoL outcomes in fatigue and social functioning, and those with progressive courses had a worse outcome in general health (reference: complete remission). Patients in treatment showed worse HRQoL outcomes in five domains compared to those not in treatment at the time of the survey. With "diagnosed in the last six months" as the reference, patients with a diagnosis more than 5 years ago reported better general health, social, and emotional functioning. Patients diagnosed between 1 and 2 years ago reported better outcomes in dyspnea and general health; those diagnosed between 2 and 5 years ago had better general health. No differences were found in physical functioning, pain, and fatigue (Table 3).

\subsubsection{Treatments Received}

Treatments and combinations of treatments were significantly associated with HRQoL in two domains (reference: surgery alone). Patients who had received chemotherapy $(\mathrm{CT})+$ surgery + radiotherapy $(\mathrm{RT})$ showed poorer physical functioning and fatigue. Patients with surgery $+\mathrm{CT}$ experienced worse physical functioning. Patients with CT alone reported worse fatigue Table 3). 


\section{Discussion}

\subsection{Results in Context}

Sarcoma patients and survivors are severely restricted in their health-related quality of life. Compared to the general population, role and social functioning are particularly strongly limited. The proportion of people with clinically important restrictions is consistently high, with a majority of patients reporting limitations in emotional, physical, and role functioning. The highest symptom burden is observed in pain, fatigue, and dyspnea. In comparison, the burden is rather low in gastrointestinal symptoms with the exception of GIST patients, who often receive tyrosine kinase inhibitors as long-term medication. The results of previous papers generally fit in well with the results of the PROSa study. Studies in more focused populations reported limitations in participation in daily life [22], physical limitations [23,24], and emotional stress [24,26,30]. Similar observations were also made regarding symptom burden, including fatigue [28,31], pain [25,30-32], shortness of breath [25-27], and insomnia [26,28].

The statistical analysis shows the association of socio-demographic factors as well as tumor and treatment-related factors with different HRQoL domains. Particularly noteworthy are the associations with socio-demographic factors that can be observed across almost all domains. While the association with age and gender is observed in almost all HRQoL studies [33], the positive correlation between HRQoL and early retirement/old age pension is not a general finding in oncology [34,35]. It seems possible that with the removal of occupational demands, mental and physical capacities are released, which ultimately has a positive effect on HRQoL. An indication of this is the strongly diminished role functioning in the study patients. Bone sarcomas occur particularly often in early adulthood, when the daily pressures in terms of work and child care are more prominent than in retirement.

Noteworthy as well and a strong indicator for the high heterogeneity of the disease are the differences we found in the most common sarcoma groups and tumor sites. All three bone sarcoma entities performed worse in the majority of analyzed domains than patients with liposarcomas, which we chose as a reference. Differences were observed within soft tissue sarcomas as well here patients with undifferentiated/unclassified sarcomas showed worse outcomes in some functioning scales. The soft tissue sarcoma groups are in part strongly diversified in itself-especially undifferentiated/unclassified as well as fibro/myofibroblastic sarcomas so that a more detailed analysis might show even more nuanced differences. The same is true for our analysis of different tumor sites. Patients with sarcomas of the upper extremities performed better than those with a tumor of the lower extremities. This might be in part an effect of the different functional restrictions related to the location of the tumor. However, differences were found in symptom scales as well especially in pain. Patients with tumors of the head and neck showed better outcomes in most functional scales and pain, and results for sarcomas of the trunk (thorax, abdomen) pointed in the same direction. The large study by von Eck et al in a population of Dutch sarcoma survivors found fewer differences with regard to tumor location than our analysis. The most affected group in that study (patients with sarcomas of the axial skeleton) cannot be directly depicted in our analysis as we chose a different classification system, but we see no indication that this group (if chosen) would have performed better in our analysis. Other differences between the study results-especially with regard to the results of patients with sarcomas of the lower extremities-are not easy to explain. It might be the case that the different study populations had an influence here [29].

Tumor-related factors (tumor size, grading) showed significant correlations in only a few or no HRQoL domains. This may be due to the fact that such information is not collected for all entities or in all situations. Another plausible hypothesis is that we included variables (tumor recurrence, disease status, treatment status) in the model that lie on the direct causal paths of the tumor-related factors und thus reduce the strength of the effect. We suspect the same mechanism with regard to treatment status, combined treatments, and metastasis by the time of study inclusion. The causal mechanisms and confounding structures are quite complex in this case (see supplement DAG). Treatment status 
and tumor recurrence are the two variables that showed significant and relevant associations in the most domains.

With regard to time since diagnosis, we found HRQoL improvements in only some domains, and these were mostly in the longer-term time spans. These results are to be interpreted with caution. The overall majority of our study participants were included during hospital/ practice visits. In survivors, clinical contacts become less frequent over time. It's very likely that we have a sick survivor bias and included selected patients with more severe disease courses. That said, it is a worthwhile research question if our findings could be repeated in less selected populations.

\subsection{Strengths and Limitations}

The PROSa study is to our knowledge one of the largest studies on HRQoL in sarcoma patients and survivors worldwide. Patients from 39 hospitals and practices were included. The participating centers comprehensively represent the aspects of sarcoma treatment in Germany and have a large network of referring institutions. Previously published studies were often limited to subgroups specified by type, localization, or treatment, or were conducted in single centers and therefore did not allow inter group comparisons. Our analysis can provide an overview of the sarcoma patient population as it is presented at our study centers. The possible exception are sarcomas of the skin, which are often treated solely within the dermatology departments.

Since sarcomas present in an extremely heterogeneous clinical picture, the analysis of subtypes is even more necessary than usual. We were able to identify specific HRQoL issues in some of these groups, but we suspect that specific relations for example, concerning the influence of treatments or histological subtypes can only become visible in even more detailed analysis. It should also be noted that a symptom-specific questionnaire for sarcomere patients did not exist at the time of study execution. It can be assumed that in particular the limitations in the functioning scales are caused by a broad spectrum of site, tumor and treatment specific factors which present in variety of ways that could only be superficially captured by the generic questions of the European Organization for Research and Treatment of Cancer Quality of Life Questionnaire (EORTC QLQ-C30) [36,37].

The present study had a cross-sectional design. Causal conclusions are therefore not directly possible. It is also subject to selection bias. We see this possibility mainly on the level of the study centers. The majority of our patients were recruited in university hospitals and/or specialized centers and might so not representative for all sarcoma patients. Selection bias is also possible at the patient level. Here we suspect a sick survivor bias, as healthy survivors have less frequent contact with our recruiting study centers. The non-participant analysis, however, does not indicate any major systematic errors in that respect. The non-participant analysis is subject to the reservation that we have not been able to determine the exact number of non-participants and not every study center reported medical data on them. The possibility of undetected systematic confounding is inherent in observational studies, but we were able to measure a broad variety of potentially confounding variables.

\section{Patients and Methods}

To reach out to the broadest possible range of sarcoma treating facilities, data collection was preceded by extensive networking involving patient representatives, research communities, and professional societies. The prospective PROSa (Burden and medical care of sarcoma in Germany: Nationwide cohort study focusing on modifiable determinants of Patient-Reported Outcome measures in Sarcoma patients) cohort study (www.uniklinikum-dresden.de/prosastudie) was conducted nationwide between September 2017 and February 2019 in 39 study centers (NCT03521531; ClinicalTrials.gov). Of those 8 were office-based practices, 22 hospitals of maximum care and 9 other hospitals.

For the present analysis, cross-sectional data of adult patients and survivors with histologically proven sarcoma of any entity were analyzed (see Table S1). We excluded persons who were mentally or linguistically unable to complete questionnaires. Only participants with HRQoL data were analyzed. 
Eligible patients and survivors were asked to participate at the participating study centers during visits (treatment, diagnose, aftercare) and sometimes by phone or letter. Participation required consent. The study was approved by the ethics committees of the Technical University of Dresden (EK1790422017) and the participating centers [38].

Data was collected by the study coordination center at University Hospital Dresden. HRQoL data and socio-demographic data were sent by the participants to the study coordination center by mail or online. Clinical information was submitted to the study coordination center online by the participating study centers using documentation forms. Data collection was performed using REDCap (Vanderbilt University, Nashville, United States) electronic data capture tools hosted at Technical University Dresden [39].

\subsection{Variables}

HRQoL was measured by means of the European Organization for Research and Treatment of Cancer Quality of Life Questionnaire (EORTC QLQ-C30) [40] This instrument measures global quality of life as well as 5 functioning and 9 symptom scales in values from 0 to $100 \mathrm{~s}$. Higher values indicate a better quality of life for the functioning scales and a higher symptom burden for the symptom scales. Socioeconomic status (SES) was assessed using the Winkler Index [41]. The Winkler Index is a composite score which covers and quantifies three dimensions of SES: income, education and occupational prestige. On a scale of 3 to 21, a lower score means a lower SES.

In addition to age and gender, the clinical variables sarcoma type (undifferentiated/ unclassified, fibro/myofibroblastic, liposarcoma, leiomyosarcoma, osteosarcoma, chondrosarcoma, synovial sarcoma, Ewing sarcoma, GIST, other), tumor location (abdomen/retroperitoneum, thorax, pelvis, lower limbs, upper limbs, head \& neck, back/spine, other sites (Table S2)), grading (G1, G2/G3), tumor size at diagnosis (T1, T2-T4, unknown + not applicable), tumor aggressiveness (locally aggressive + rarely metastatic, malignant), metastases up to the time of inclusion (yes, no, unknown), comorbidities at inclusion $(0,1,2,3,>3)$, disease status (complete remission, partial remission + stable disease, progression, unknown), treatment intention (palliative, curative), treatment status (yes, no), performed treatments (surgery alone, surgery + chemotherapy $(\mathrm{CT})$, surgery + radiotherapy $(\mathrm{RT})$, surgery $+\mathrm{RT}+$ CT, CT alone, other), time since diagnosis ( $<6$ months, $6<12$ months, $12<24$ months, $24<60$ months, 60 months or more), and tumor recurrence (yes, no, unknown) were evaluated (for a tabular overview of the variables see Table 1).

\subsection{Statistics}

Continuous variables were evaluated by mean and standard deviation (SD) if normally distributed and by median and interquartile range (IQR) if not. Categorical variables were presented with absolute and relative frequencies. To contextualize results, an age and gender standardized comparison with reference values of the German normal population was performed [42]. The relevance of the differences was evaluated using reference values from Cocks and Osoba [43,44]. With these reference values, each scale difference can be classified as "small", "moderate" and "large" (Osoba) or "trivial", "small", "medium" and "large" (Cocks).

We also reported stratified by sarcoma type and treatment intention the proportion of patients with clinically important symptoms and limitations (CIS $+\mathrm{L}$ ) in the HRQoL domains using the thresholds of Giesinger et al. According to Giesinger et al. the concept of clinically important symptoms and limitations was developed to meet "the need for well-defined, valid thresholds for the absolute scores on the EORTC QLQ-C30 based on external criteria reflecting the clinical importance of a health problem. Clinical importance is defined as any aspect of a health problem that makes it relevant for the clinical encounter" [45].

We used a flow chart to report on study participation. A non-participant analysis was performed to estimate possible selection bias. The data of the non-participants, participants without HRQoL and the evaluated population were compared (Table S3). 
Eight selected domains of EORTC QLQ-C30 (global quality of life, physical, social, emotional and role functioning, pain, fatigue, shortness of breath) were examined for associated factors. For this purpose, multivariable linear regressions were calculated and unstandardized regression coefficient (B), confidence intervals, p-values and $\mathrm{R}^{2}$ were evaluated for the whole model. Again, the relevance of the differences was evaluated using reference values from Cocks and Osoba [43,44].

Model variables were selected using direct acyclic graphs before analysis [46] (Figure S1).

To reduce the proportion of missing values in the SES, an imputation procedure was performed under the missing-completely-at-random assumption. The mean value of the overall index was calculated for the individual variable values of the three SES dimensions. If information on a single dimension was available in the participant's data set, the overall mean value was imputed as the individual mean value of the participant.

Categorical variables were included in the analysis using dummy variables. To avoid multicollinearity, correlations and tolerance between the model variables were calculated before regression analyses. Correlations $\geq 0.7$ and tolerance values $\leq 0.1$ indicate strong multicollinearity problems. As a result, treatment intention was not included in the model and were evaluated in a stratified analysis.

Statistical analyses were performed with SPSS V.25 (IBM Corporation, Armonk, New York, NY, USA).

\section{Conclusions}

Compared to a German population, sarcoma patients and survivors are severely restricted in their health-related quality of life. The majority of them report clinically important restrictions in role, physical, and emotional functioning. Approximately half of the patients and survivors suffer from clinically important pain, fatigue, and dyspnea. Sociodemographic factors are associated with HRQoL limitations; the observed impact of reaching old age pension/early retirement for an increase in HRQoL is to be highlighted. We found a number of indications as to how the diversity of sarcoma disease manifests itself in HRQoL. Patients with sarcomas of the upper extremities, head and neck, of the abdominal/retroperitoneal region as well as the thoracic region performed better than those with sarcomas of the lower extremities. Patients with bone sarcomas had more severe HRQoL restrictions than soft tissue sarcoma patients, but there were also differences to be observed within the various soft tissue sarcoma entities.

During treatment and aftercare, increased attention should be paid to the frequent clinically important restrictions and symptoms of sarcoma patients especially with regard to role, physical, social and emotional functioning as well as to fatigue, pain and dyspnea.

Supplementary Materials: The following are available online at http://www.mdpi.com/2072-6694/12/12/3590/s1, Table S1: Sarcoma subtypes by histology, Table S2: Construction of sarcoma location variable, Table S3: Comparison study population with study participants without questionnaire and non-participants, Figure S1: Directed acyclic graphs of potential predictive variables of HRQoL of sarcoma patients. Yellow: recorded predictive variables, grey: variables not included in the model, blue: outcome. The absence of red arrows implies a model without uncontrolled confounding.

Author Contributions: M.E. wrote the article and analyzed the data. M.E., M.K.S. and L.H. developed questionnaires and study design. J.S. and M.K.S. developed the conception of the study and supervised with M.B. the work throughout the whole study. E.W. supervised development of inclusion criteria. K.A. supervised the study from a patient's perspective. M.E., M.K.S., J.S., L.H., S.R., P.H., B.K., D.A., J.J. and S.S. developed the statistical analysis plan for this paper. S.R., P.H., B.K., D.A., D.P., J.J., R.G., S.F., C.H., M.F., V.H., V.I.G., K.V., M.A., J.W., K.-D.S. and M.K.S. were responsible for the recruitment of patients or recruited patients directly. All authors have revised the manuscript critically and approved the published version. All authors have read and agreed to the published version of the manuscript.

Funding: The PROSa study was funded by the German Cancer Aid (No. 111713).

Acknowledgments: Without the support of the following individuals, the conduct of our study would not have been possible. We are very grateful for the support received. Christiane Noack, Gina Lehmann, Christine Peikert, Luise Mütze, Armina Kalirad, Alexander Kohler, Jessica Pablik, Gustavo B. Baretton, Pauline Wimberger, Maria Eberlein-Gonska, Mechthild Krause, Beate Hornemann, Ralf-Thorsten Hoffmann, Chiara Valentini, Christina Jentsch, Fabian Lohaus, Gerhard Ehninger, Hagen Fritsche, Martin Rößler, Olaf Schoffer, Freya Trautmann, Susan Vogl, Diana Löscher, Michael Laniado, Johanna Kirchberg, Christine Griebsch (University 
Hospital Dresden), Lothar Müller, Carsten Janssen, Johanna Jansen, Kim-Aline Kraft (Oncological medical practice UnterEms), Gerdt Hübner, Renate Günther (ohO Ostholstein), Torsten Kluba, Kerstin Spranger, Nicole Thoms-Ruckau, Juliane Gehre (Hospital Dresden-Friedrichstadt), Stefan Fuxius, Andreas Karcher, Tanja Löschner (Oncological medical practice Heidelberg), Claudia Fendrich, Monika Mathew, Franka Menge (University Hospital Mannheim), Ralph Naumann, Katja Schneider (St. Marien- Hospital Siegen), Ulrich Kaiser, Monika Morban (St. Bernward Hospital Hildesheim), Martina Popp (Helios Hospital Emil von Behring), Simone Micheel (Helios Hospital Bad Saarow), Michael Ehrsam (Oncological medical practice Schwarzwald Alb), Henriette Golcher, Katrin Hindel, Nikolaos Vassos, Maximilian Brunner, Justus Baecker, Katja Fechner, Thomas Förtsch, Claudia Handtrack, Felix Wiesmüller, Veronika Zver, Stefania Puta, Sabine Semrau, Rainer Fietkau, Matthias Beckmann, Judith Bürner, Lena Kellner, Dorotha Lubgan (University Hospital Erlangen), Peter Reichardt, Annette Reichhardt, Per-Ulf Tunn (Helios Hospital Berlin-Buch), Stephan Mose, Mohammed Reda Al-Omar (Schwarzwald-Baar Hospital), Gaby Horstmann, Tanja Trarbach (Hospital Wilhelmshaven), Thomas Wölfel, Manuel Baltzereit, Sergio Armando Zapata Bonilla, Karl Friedrich Kreitner (University Hospital Mainz), Annegret Kunitz, Anne Leopold (Vivantes Berlin), Wolfram Knoefel, Hany Ashmawy (University Hospital Düsseldorf), Christian A. Schmidt, Jeanette Bahr (University Hospital Greifswald), Johannes Mohm, Gabriele Prange-Krex, Catrin Krüger (Oncological medical practice Dres. Mohm, Prange-Krex Dresden), Susanne Pfitzner-Dempfle, Marion Sollgan (Oncological medical practice Kaiserslautern), Stephanie Erdmann, Dorothea Zaech (Oncological medical practice Dres. Verpoort \& Wierecky), Ursula Vehling-Kaiser, Ana Hoffmann, Maxi Hofmann (H.O.T. Landshut), Gerlinde Egerer, Martina Gronkowski (University Hospital Heidelberg), Hans-Günter Derigs, Diana Isabella Dmytrow, Claudia Bund (Hospital Frankfurt Höchst), Ulrich Kneser, Amir Bigdeli, Steffen Nolte (BG Hospital Ludwigshafen), Claus-Henning Köhne, Ute Schubert (Hospital Oldenburg), Christiane Reinert, Anke Schlenska-Lange, Nadia Maguire (Barmherzige Brüder Regensburg), Regine Mayer-Steinacker, Evelyn Mack (University Hospital Ulm), Armin Tuchscherer, Roland Ulrich, Christina Thielen, Lars Pester, Cäcilia Kaul (University Hospital Cologne), Carsten Schlickewei, Matthias Primel (University Hospital Hamburg Eppendorf), Roswitha Rausch, Thomas Hawighorst (Hospital Fulda), Claudia Bosch, Christoph Anthuber (Hospital Starnberg), Simone Hettmer, Barbara Wirth, Holger Bannasch (University Hospital Freiburg), Nicola Gökbuget, Lena Schütz (University Hospital Frankfurt), Matthias Grube, Tanja Hofmann, Christian Stroszczynski (University Hospital Regensburg), Carolin Tonus, Nadine Sykosch (Asklepios Hospital St. Georg Hamburg), Inga Kreisel (University Hospital Göttingen), Jalid Sehouli (Charité Berlin), Uta Dirksen (University Hospital Essen), Markus Wartenberg (German Sarcoma Foundation).

Conflicts of Interest: S.S. received lecture fees from Lilly, BMS, Boehringer-Ingelheim, and Pfizer, all outside of this work. J.S. received consulting fees from Novartis, Sanofi, A.L.K. and Lilly, all outside of this work. J.J. received fees from Lilly and Boehringer Ingelheim, all outside of this work. L.H. received fees from SERVIER, outside of this work. D.A. received lecture fees from Lilly and Implantcast, all outside of this work. M.A. received fees from Blueprint Medicines for conducting contract clinical trials and reimbursement of conference attendance fees from Blueprint Medicines, PharmaMar, and Lilly, all outside of this work. M.F. was reimbursed for congress attendance fees and travel and accommodation expenses by PharmaMar and Lilly, all outside of this work. D.P. received fees for consulting services from Lilly, PharmaMar, Roche, and fees for lectures from Lilly and PharmaMar, all outside of this work. S.F. received reimbursements for congress attendance fees from PharmaMar, all outside of this work. V.I.G. received reimbursements of congress attendance fees and travel and accommodation expenses from Novartis and Lilly, all outside this work. M.K.S. received research funding from PharmaMar and Novartis, all outside of this work. M.E., C.H., B.K., K.-D.S., K.A., M.B., V.H., J.W., R.G., E.W., P.H. \& S.R. declare that no conflicts of interest exist.

\section{References}

1. Ressing, M.; Wardelmann, E.; Hohenberger, P.; Jakob, J.; Kasper, B.; Emrich, K.; Eberle, A.; Blettner, M.; Zeissig, S.R. Strengthening health data on a rare and heterogeneous disease: Sarcoma incidence and histological subtypes in Germany. BMC Public Health 2018, 18, 1-11. [CrossRef]

2. Stiller, C.; Trama, A.; Serraino, D.; Rossi, S.; Navarro, C.; Chirlaque, M.; Casali, P. Descriptive epidemiology of sarcomas in Europe: Report from the RARECARE project. Eur. J. Cancer 2013, 49, 684-695. [CrossRef] [PubMed]

3. Fletcher, C.D.M. World Health Organization. International Agency for Research on Cancer (Hrsg.): WHO Classification of Tumours of Soft Tissue and Bone, 4th ed.; IARC Press: Lyon, France, 2013.

4. Casali, P.; Abecassis, N.; Bauer, S.; Biagini, R.; Bielack, S.; Bonvalot, S.; Boukovinas, I.; Bovee, J.V.M.G.; Brodowicz, T.; Broto, J.; et al. Soft tissue and visceral sarcomas: ESMO-EURACAN Clinical Practice Guidelines for diagnosis, treatment and follow-up. Ann. Oncol. 2018, 29, iv51-iv67. [CrossRef] [PubMed]

5. Biermann, J.S.; Chow, W.; Reed, D.R.; Lucas, D.; Adkins, D.R.; Agulnik, M.; Benjamin, R.S.; Brigman, B.; Budd, G.T.; Curry, W.T.; et al. NCCN Guidelines Insights: Bone Cancer, Version 2.2017. J. Natl. Compr. Cancer Netw. 2017, 15, 155-167. [CrossRef] [PubMed]

6. Nishida, T.; Blay, J.-Y.; Hirota, S.; Kitagawa, Y.; Kang, Y.-K. The standard diagnosis, treatment, and follow-up of gastrointestinal stromal tumors based on guidelines. Gastric Cancer 2016, 19, 3-14. [CrossRef] 
7. Neuwirth, M.G.; Song, Y.; Sinnamon, A.J.; Fraker, D.L.; Zager, J.S.; Karakousis, G.C. Isolated Limb Perfusion and Infusion for Extremity Soft Tissue Sarcoma: A Contemporary Systematic Review and Meta-Analysis. Ann. Surg. Oncol. 2017, 24, 3803-3810. [CrossRef] [PubMed]

8. Issels, R.D.; Lindner, L.H.; Verweij, J.; Wessalowski, R.; Reichardt, P.; Wust, P.; Vujaskovic, Z. Effect of Neoadjuvant Chemotherapy Plus Regional Hyperthermia on Long-term Outcomes Among Patients with Localized High-Risk Soft Tissue Sarcoma: The EORTC 62961-ESHO 95 Randomized Clinical Trial. JAMA Oncol. 2018, 4, 483. [CrossRef]

9. Soomers, V.L.M.N.; Husson, O.; Young, R.; Desar, I.; Van Der Graaf, W. The sarcoma diagnostic interval: A systematic review on length, contributing factors and patient outcomes. ESMO Open 2020, 5, e000592. [CrossRef]

10. Kang, S.; Kim, H.-S.; Han, I. Unplanned Excision of Extremity Soft Tissue Sarcoma in Korea: A Nationwide Study Based on a Claims Registry. Duan Z (Hrsg.). PLoS ONE 2015, 10, e0134354.

11. Traub, F.; Griffin, A.M.; Wunder, J.S.; Ferguson, P.C. Influence of unplanned excisions on the outcomes of patients with stage III extremity soft-tissue sarcoma: Outcome of Unplanned Excisions in STS. Cancer 2018, 124, 3868-3875. [CrossRef]

12. Available online: https://www.krebsgesellschaft.de/zertkomm-protokolle.html?file=files/dkg/deutschekrebsgesellschaft/content/pdf/Zertifizierung/Protokolle_Zertkomm/SAR_Protokoll_190225.pdf\&cid=61328 (accessed on 30 November 2020).

13. Dangoor, A.; Seddon, B.; Gerrand, C.; Grimer, R.J.; Whelan, J.S.; Judson, I. UK guidelines for the management of soft tissue sarcomas. Clin. Sarcoma Res. 2016, 6, 1-26. [CrossRef] [PubMed]

14. Available online: https://www.sarkome.de/ (accessed on 30 November 2020).

15. Available online: https://euracan.ern-net.eu (accessed on 30 November 2020).

16. Meropol, N.J.; Egleston, B.L.; Buzaglo, J.S.; Benson, A.B.; Cegala, D.J.; Diefenbach, M.A.; Fleisher, L.; Miller, S.M.; Sulmasy, D.P.; Weinfurt, K.P.; et al. Cancer patient preferences for quality and length of life. Cancer 2008, 113, 3459-3466. [CrossRef] [PubMed]

17. Higginson, I.J.; Gomes, B.; Calanzani, N.; Gao, W.; Bausewein, C.; A Daveson, B.; Deliens, L.; Ferreira, P.L.; Toscani, F.; Gysels, M.; et al. Priorities for treatment, care and information if faced with serious illness: A comparative population-based survey in seven European countries. Palliat. Med. 2014, 28, 101-110. [CrossRef] [PubMed]

18. Winnette, R.; Hess, L.M.; Nicol, S.J.; Tai, D.F. Copley-Merriman C: The Patient Experience with Soft Tissue Sarcoma: A Systematic Review of the Literature. Patient Patient-Cent. Outcomes Res. 2016, 10, 153-162. [CrossRef]

19. McDonough, J.; Eliott, J.; Neuhaus, S.J.; Reid, J.; Butow, P. Health-related quality of life, psychosocial functioning, and unmet health needs in patients with sarcoma: A systematic review. Psycho Oncol. 2019, 28, 653-664. [CrossRef]

20. Coens, C.; Van Der Graaf, W.T.; Blay, J.Y.; Chawla, S.P.; Judson, I.; Sanfilippo, R.; Lugowska, I. Health-related quality-of-life results from PALETTE: A randomized, double-blind, phase 3 trial of pazopanib versus placebo in patients with soft tissue sarcoma whose disease has progressed during or after prior chemotherapy-a European Organization for res: HRQoL of Pazopanib in Pretreated STS. Cancer 2015, 121, 2933-2941.

21. Poveda, A.; López-Pousa, A.; Martín, J.; Del Muro, J.G.; Bernabé, R.; Casado, A.; Balañá, C.; Sanmartín, O.; Menéndez, M.D.; Escudero, P.; et al. Phase II Clinical Trial with Pegylated Liposomal Doxorubicin (CAELYX ${ }^{\circledR} /$ Doxil\&reg;) and Quality of Life Evaluation (EORTC QLQ-C30) in Adult Patients With Advanced Soft Tissue Sarcomas: A study of the Spanish Group for Research in Sarcomas (GEIS). Sarcoma 2005, 9, 127-132. [CrossRef]

22. Schreiber, D.; Bell, R.S.; Wunder, J.S.; O'Sullivan, B.; Turcotte, R.; Masri, B.A.; Davis, A.M. Evaluating Function and Health Related Quality of Life in Patients Treated for Extremity Soft Tissue Sarcoma. Qual. Life Res. 2006, 15, 1439-1446. [CrossRef]

23. Kwong, T.N.K.; Furtado, S.; Gerrand, C. What do we know about survivorship after treatment for extremity sarcoma? A systematic review. Eur. J. Surg. Oncol. 2014, 40, 1109-1124. [CrossRef]

24. Saebye, C.; Fugloe, H.M.; Nymark, T.; Safwat, A.; Petersen, M.M.; Baad-Hansen, T.; Krarup-Hansen, A.; Keller, J. Factors associated with reduced functional outcome and quality of life in patients having limb-sparing surgery for soft tissue sarcomas-A national multicenter study of 128 patients. Acta Oncol. 2017, 56, 239-244. [CrossRef] 
25. Gough, N.J.; Smith, C.; Ross, J.R.; Riley, J.; Judson, I. Symptom Burden, Survival and Palliative Care in Advanced Soft Tissue Sarcoma. Sarcoma 2011, 2011, 1-8. [CrossRef] [PubMed]

26. Gough, N.; Koffman, J.; Ross, J.R.; Riley, J.; Judson, I. Symptom Burden in Advanced Soft-Tissue Sarcoma. J. Pain Symptom Manag. 2017, 53, 588-597. [CrossRef] [PubMed]

27. Reichardt, P.; Leahy, M.; Del Muro, X.G.; Ferrari, S.; Martin, J.; Gelderblom, H.; Wang, J.; Krishna, A.; Eriksson, J.; Staddon, A.; et al. Quality of Life and Utility in Patients with Metastatic Soft Tissue and Bone Sarcoma: The Sarcoma Treatment and Burden of Illness in North America and Europe (SABINE) Study. Sarcoma 2012, 2012, 740279. [CrossRef]

28. Chan, A.; Lim, E.; Ng, T.; Shih, V.; Quek, R.; Cheung, Y.T. Symptom burden and medication use in adult sarcoma patients. Support. Care Cancer 2015, 23, 1709-1717. [CrossRef] [PubMed]

29. Van Eck, I.; Hollander, D.D.; Desar, I.M.; Soomers, V.L.; Van De Sande, M.A.; De Haan, J.J.; Verhoef, C.; Vriens, I.J.; Bonenkamp, J.J.; Van Der Graaf, W.T.; et al. Unraveling the Heterogeneity of Sarcoma Survivors' Health-Related Quality of Life Regarding Primary Sarcoma Location: Results from the SURVSARC Study. Cancers 2020, 12, 3083. [CrossRef]

30. Sachsenmaier, S.; Ipach, I.; Kluba, T. Quality of Life, Physical and Mental Status and Contentment of Patients with Localized Soft Tissue or Bone Sarcoma: A Questionnaire Analysis. Orthop. Rev. 2015, 7, 7. [CrossRef]

31. Van Der Geest, I.; Knoop, H.; Veth, R.P.; Schreuder, H.W.; Bleijenberg, G. High fatigue scores before and after surgical treatment of bone and soft tissue tumors. Exp. Ther. Med. 2013, 5, 205-208. [CrossRef]

32. Trautmann, F.; Singer, S.; Schmitt, J. Patients with soft tissue sarcoma comprise a higher probability of comorbidities than cancer-free individuals. A secondary data analysis. Eur. J. Cancer Care 2017, 26, e12605. [CrossRef]

33. Schwarz, R.; Hinz, A. Reference data for the quality of life questionnaire EORTC QLQ-C30 in the general German population. Eur. J. Cancer 2001, 37, 1345-1351. [CrossRef]

34. Hechtner, M.; Eichler, M.; Wehler, B.; Buhl, R.; Sebastian, M.; Stratmann, J.; Schmidberger, H.; Gohrbandt, B.; Peuser, J.; Kortsik, C.; et al. Quality of Life in NSCLC Survivors-A Multicenter Cross-Sectional Study. J. Thorac. Oncol. 2019, 14, 420-435. [CrossRef]

35. Tomaszewski, K.A.; Püsküllüoğlu, M.; Biesiada, K.; Bochenek, J.; Ochenduszko, S.; Tomaszewska, I.M.; Krzemieniecki, K. Main influencing factors and health-related quality of life issues in patients with oesophago-gastric cancer-As measured by EORTC tools. Wspótczesna Onkol. 2013, 3, 311-316. [CrossRef] [PubMed]

36. Husson, O.; Hollander, D.D.; Van Der Graaf, W.T.A. The complexity of assessing health-related quality of life among sarcoma patients. Qual. Life Res. 2020, 29, 2613-2614. [CrossRef] [PubMed]

37. Gough, N.; Koffman, J.; Ross, J.R.; Riley, J.; Judson, I. Does palliative chemotherapy really palliate and are we measuring it correctly? A mixed methods longitudinal study of health related quality of life in advanced soft tissue sarcoma. PLoS ONE 2019, 14, e0210731. [CrossRef] [PubMed]

38. Eichler, M.; Schmitt, J.; Schuler, M.K. Die Dauer von Ethikvoten in Deutschland-Am Beispiel einer nicht-interventionellen Beobachtungsstudie mit 44 teilnehmenden Zentren (PROSa). Z. Evidenz Fortbild. Qual. Gesundh. 2019, 146, 15-20. [CrossRef] [PubMed]

39. Harris, P.A.; Taylor, R.; Thielke, R.; Payne, J.; Gonzalez, N.; Conde, J.G. Research electronic data capture (REDCap)-A metadata-driven methodology and workflow process for providing translational research informatics support. J. Biomed. Inform. 2009, 42, 377-381. [CrossRef]

40. Aaronson, N.K.; Ahmedzai, S.; Bergman, B.; Bullinger, M.; Cull, A.; Duez, N.J.; Filiberti, A.; Flechtner, H.; Fleishman, S.B.; De Haes, J.C.; et al. The European Organization for Research and Treatment of Cancer QLQ-C30: A Quality-of-Life Instrument for Use in International Clinical Trials in Oncology. J. Natl. Cancer Inst. 1993, 85, 365-376. [CrossRef]

41. Lampert, T.; Kroll, L.E.; Müters, S.; Stolzenberg, H. Messung des sozioökonomischen Status in der Studie Gesundheit in Deutschland aktuell (GEDA). Bundesgesundheitsblatt Gesundh. Gesundh. 2013, 56, 131-143. [CrossRef]

42. Nolte, S.; Liegl, G.; Petersen, M.; Aaronson, N.K.; Costantini, A.; Fayers, P.; Groenvold, M.; Holzner, B.; Johnson, C.; Kemmler, G.; et al. General population normative data for the EORTC QLQ-C30 health-related quality of life questionnaire based on 15,386 persons across 13 European countries, Canada and the Unites States. Eur. J. Cancer 2019, 107, 153-163. [CrossRef] 
43. Cocks, K.; King, M.T.; Velikova, G.; St-James, M.M.; Fayers, P.M.; Brown, J.M. Evidence-Based Guidelines for Determination of Sample Size and Interpretation of the European Organisation for the Research and Treatment of Cancer Quality of Life Questionnaire Core 30. J. Clin. Oncol. 2011, 29, 89-96. [CrossRef]

44. Osoba, D.; Rodrigues, G.; Myles, J.; Zee, B.; Pater, J. Interpreting the significance of changes in health-related quality-of-life scores. J. Clin. Oncol. 1998, 16, 139-144. [CrossRef]

45. Giesinger, J.; Loth, F.L.; Aaronson, N.K.; Arraras, J.I.; Caocci, G.; Efficace, F.; Groenvold, M.; Van Leeuwen, M.; Petersen, M.A.; Ramage, J.; et al. Thresholds for clinical importance were established to improve interpretation of the EORTC QLQ-C30 in clinical practice and research. J. Clin. Epidemiol. 2020, 118, 1-8. [CrossRef] [PubMed]

46. Textor, J.; Hardt, J.; Knüppel, S. DAGitty: A graphical tool for analyzing causal diagrams. Epidemiology 2011, 22, 745. [CrossRef] [PubMed]

Publisher's Note: MDPI stays neutral with regard to jurisdictional claims in published maps and institutional affiliations.

(C) 2020 by the authors. Licensee MDPI, Basel, Switzerland. This article is an open access article distributed under the terms and conditions of the Creative Commons Attribution (CC BY) license (http://creativecommons.org/licenses/by/4.0/). 